\title{
Water wave diffraction by a cylinder array. Part 1. Regular waves
}

\author{
By C. O. G. OHL†, R. EATOCK TAYLOR, P. H. TAYLOR \\ AND A. G. L. BORTHWICK
}

Department of Engineering Science, University of Oxford, Parks Road, Oxford OX1 3PJ, UK

(Received 15 June 2000 and in revised form 14 December 2000)

Diffraction of regular waves by arrays of vertical bottom-mounted circular cylinders is investigated using theoretical, computational, and experimental methods. Experiments in an offshore wave basin are designed to measure free surface elevation $\eta$ at multiple locations in the vicinity of a multi-column structure subjected to regular waves of frequency $0.449<k a<0.524$ and steepness $0.122<k A<0.261$, where $k$ is the wavenumber, $a$ the cylinder radius and $A$ the wave amplitude. Results from regular wave data analysis for first-order amplitudes are compared with those from analytical linear diffraction theory, which is shown to be accurate for predicting incident waves of low steepness. Second- and third-order terms are also estimated from the measured time series, and the effects near a second-order near-trapping frequency are compared to semi-analytical second-order diffraction theory. Linear diffraction theory is shown to be very accurate at predicting the global surface elevation features, even for waves of high steepness. However, violent events and significant nonlinear interactions, including breaking induced by wave scattering, have been observed. Furthermore, second-order near-trapping was observed to affect the magnitude of local free surface oscillations as well as scattered far-field radiation.

\section{Introduction}

Two closely related problems considered in the design of offshore platforms are 'upwelling' and 'runup'. Upwelling is the local modification (and possible amplification) of the incident surface wave in the vicinity of the structure, whereas runup is the local modification immediately adjacent to a given surface-piercing element, associated with fluid flow on the surface of the body. These phenomena can cause the local wave elevations to be significantly greater than that of the undisturbed incident wave and are of particular interest due to the growing number of large diameter, multi-column offshore structures.

The reason for predicting upwelling and runup is the necessity for designers to maintain a sufficient air gap, which is the vertical distance from the underside of the platform deck to the wave crest in extreme design storm conditions. While this clearance must be sufficient that the wave does not strike the lower deck, it must also be minimized in order to avoid raising the centre of gravity and exposed vertical surface area of the platform, which may affect the wind loading and will increase the wind overturning moments. Keeping the air gap of a tension leg platform as small as possible also has the desirable effect of minimizing the weight of the structure.

$\dagger$ Present address: c/o Human Resources, Costain Limited, Costain House, Nicholson's Walk, Maidenhead, Berkshire SL6 1LN, UK. 
Aside from elevation effects, upwelling alters the local wave kinematics near the platform. If such an alteration results in increased horizontal velocity, drag forces may be amplified on low-level appendages such as conductors and risers. Damage to the lowest decks of semi-submersible platforms (Eatock Taylor \& Wu 1997) and gravity platforms (Swan, Taylor \& Van Langen 1997) has been reported within the offshore industry. In addition, experiments on various multi-column structures (Owen \& Padilla Perez 1987 and Swan et al. 1997) have demonstrated higher than expected local wave elevations, which suggest the presence of significant nonlinearities. The presence of significant runup around the columns and upwelling beneath the centre of the platform deck was also observed in model tests on the Brent Bravo GBS (Gravity Based Structure) undertaken by Swan et al. (1997); the main effect was in high waves (large steepness) of high frequency (short wavelength).

A possible contributing factor giving rise to high localized wave elevations is the phenomenon known as near-trapping. This has been shown theoretically to occur at critical frequencies dependent upon the geometry of the structure (cylinder diameter and spacing) relative to the wavelength. Discussed in more detail below, the terminology is based upon the similarity to trapping of waves near a body in a long narrow channel: at specific incident wave frequencies, there is no radiation of wave energy to infinity. This is closely related to the well-known phenomenon of edge waves at a plane sloping beach, as identified by Stokes (1846).

\subsection{Diffraction theory}

Wave-body interaction analysis is a three-dimensional, fully nonlinear problem, which, even in regular seas, unsurprisingly remains unsolved. There have been recent attempts to approximate the solution, for example using boundary element (Ferrant 1996 and Celebi, Kim \& Beck 1998) and finite element (Ma, Wu \& Eatock Taylor 2000) methods, but these are still incomplete. However, given certain assumptions and simplification, low-order analytical models have been derived. If the typical dimension (i.e. column diameter) is large compared with the surface wave amplitude and wavelength, then viscous effects can be neglected and diffraction effects are dominant. In addition, the flow may be assumed to be incompressible and irrotational, and surface tension effects assumed to be negligible. These last three assumptions imply that the flow field can be represented by a scalar velocity potential, satisfying the Laplace equation within the fluid domain. While there is an implicit time dependence in the velocity potential, the problem is often studied in the frequency domain, which reduces computation time and eases analytical progress. Through a perturbation expansion, the potential may be expressed as the sum of linear and higher-order components associated with powers of some small expansion parameter. The potential may then be solved at each order successively by using the lower-order solutions to generate the boundary conditions necessary to solve for the higher-order components. Comprehensive reviews of analytical and numerical linear diffraction analyses have been undertaken by Yeung (1982) and Mei (1983).

The primary obstacle in developing higher-order diffraction theory in the frequency domain is the inhomogeneous free surface boundary condition, which must be addressed at second order. This problem requires integration of an equation over the entire free surface, containing quadratic products of the first-order velocity potential and its derivatives.

Kriebel (1990) developed a second-order solution based on a velocity potential decomposition for the diffraction of a monochromatic wave (a regular wave of single frequency component) in the presence of a bottom-mounted, circular cylinder. Kriebel 
predicted that the maximum second order runup on the cylinder exceeded that from linear theory by more than $50 \%$ in cases of steep waves where $k A \geqslant 0.25$ ( $k$ and $A$ are the wavenumber and wave amplitude, respectively). Subsequently, Kriebel (1992) conducted laboratory experiments to measure wave runup around a large-diameter, vertical circular cylinder in monochromatic nonlinear waves. These confirmed that the second order theory explains a significant portion of the nonlinear runup.

Malenica \& Molin (1995) used eigenfunction expansions and an integral equation technique to develop expressions for the third-order velocity potential for a vertical bottom-mounted circular cylinder in regular waves. However, Malenica \& Molin only considered velocity potential components relevant to the calculation of third-order forces and did not develop any expressions for the third-order diffracted free surface elevation. While first- and second-order results were verified by comparison with the free surface elevation calculations of Kim \& Yue (1989), third-order force calculations were compared with experimental data (Moe 1993) for a single truncated cylinder. Second-order loads measured during these experiments showed very close agreement with calculated values. However, the third-order force measurements showed significant scatter, causing comparison with the theoretical values to be inconclusive. Subsequent experimental data obtained by Huseby \& Grue (1998) provided much closer agreement with Malenica \& Molin's third-order force predictions, and also with the fully nonlinear simulations of Ferrant (1996).

\subsection{Diffraction from cylinder arrays}

In the case of a wave incident on an array of bodies, computation of the velocity potential must account for both the diffraction of the incident field by each body and the multiple scattering due to the other bodies. By developing a simple formulation for this multiple scattering phenomenon, Linton \& Evans (1990) provided formulae for the first- and mean second-order forces on one cylinder in an array of bottom-mounted circular cylinders, and also an efficient method for the calculation of the first-order velocity potential (from which follows the free surface amplitude). A semi-analytical solution of second-order diffraction from an array of vertical bottom-mounted circular cylinders was presented by Malenica, Eatock Taylor \& Huang (1999). A continuation of similar approaches that were initiated independently by Huang \& Eatock Taylor (1996) and Malenica (1997), the method obtains the second-order potential by combining eigenfunction expansions with an integral representation. This allows computation of local flow characteristics, such as free surface elevation, pressure, and velocities, as well as the evaluation of third-order force components due to first- and second-order effects.

\subsection{Trapping and near-trapping of waves}

Ursell (1951) first identified trapped modes in an open channel (corresponding to a long narrow wave tank) using first-order wave theory in his study of a submerged horizontal cylinder. With the restrictions of small radius and infinite depth, he proved that, while some energy radiated to infinity, energy at discrete frequencies below a certain cutoff was trapped near the cylinder and did not radiate. Subsequently, Jones (1953) extended this to include submerged horizontal cylinders of arbitrary cross-section (i.e. not limited to small radius), but symmetrical about a vertical plane.

Evans \& Linton (1991) made numerical calculations for a vertical cylinder of rectangular cross-section placed at the centre of a long narrow wave channel (see the Appendix). With frequency dependent upon the dimensions of the rectangular cylinder relative to the channel, trapped modes were found to be antisymmetric about 
the vertical centreplane of the wave tank and either symmetric or antisymmetric about a transverse vertical plane through the cylinder centre (i.e. normal to the tank sides). Evans, Levitin \& Vassiliev (1994) subsequently proved existence of trapped modes in channels for all symmetric cross-sections.

Maniar \& Newman (1997) utilized Linton \& Evans' (1990) method to study the forces on long finite linear arrays of bottom-mounted circular cylinders in open sea, the axes of which are separated by the distance $h$. The spacing to wavelength ratio $k h$ was identified as most significant for the magnitude of free surface oscillations and scattering, although it was also dependent on the ratio $a / h$. At certain values of $k h$, large free surface oscillations in the vicinity of cylinders near the array centre decay only rather slowly with distance from the array. This corresponds to near-trapping, and leads to large forces on some of the cylinders. Maniar \& Newman (1997) made the practical suggestion that the very large free surface amplitudes and peak loads predicted at near-trapping would be substantially reduced due to viscous damping and other nonlinear effects.

Lastly, employing a semi-analytical diffraction theory for arrays of bottom-mounted circular cylinders, Evans \& Porter (1997) made a detailed investigation of neartrapping by circular arrays of vertical cylinders. Malenica et al. (1999) subsequently extended the studies of near-trapping in an array to second order. They divided the total second-order free surface elevation $\eta^{(2)}$ into the following two parts: $\eta^{(21)}$, due to quadratic products of first-order quantities, and $\eta^{(22)}$, directly from the second-order potential. For an array of four cylinders at the corners of a square of side length $2 h$ in water of depth $d=3 a$, the free surface elevation was studied along the centreline for two configurations, $a / h=0.5$ and 0.6, for which Evans \& Porter (1997) and Malenica et al. (1999) had identified first-order near-trapping at $k a=1.66$ and 2.27. While both $\eta^{(21)}$ and $\eta^{(22)}$ were large at these first-order frequencies, their relative phases were such that $\eta^{(2)}$ showed no significant response. At half the first-order near-trapping frequencies, corresponding to $k a=0.468$ and 0.600 for the case here of $d / a=3$, the first-order free surface and corresponding quadratic products, $\eta^{(1)}$ and $\eta^{(21)}$, were found to have low values. In contrast, the contribution from the secondorder potential at these frequencies for these cases led to large free surface responses: the total second-order free surface elevation, $\eta^{(2)}$, exhibited amplification as high as $\left|\eta^{(2)}\right| / k A^{2}=40$ for $a / h=0.6$. Coupled with studies of first- and second-order runup in the array as a function of $k a$ for $a / h=0.6$, these results suggest that second-order near-trapping occurs when the frequency of the second-order components is equal to the frequency of first-order near-trapping.

\subsection{Objectives}

The validation of higher-order diffraction theories has typically consisted of comparison with limited results from numerical analyses. Comparisons with experimental results have been primarily based upon the forces and/or moments experienced by structures, which are integrated quantities, as opposed to the more rigorous test of comparing free surface elevations. Where comparisons of theoretical free surface elevation to experimental measurements have been conducted, these have been for experiments involving single cylinders and small wave heights. These single cylinder cases do not constitute a test of the complex scattering problem for the interactions of multiple bodies and are of little interest for the practical design of large offshore structures. While more complex geometries such as a TLP (Tension Leg Platform) or GBS with truncated or tapering columns have been tested, these have been of sufficient complexity to render direct comparison with the most basic theories 
impossible. Furthermore, the small-amplitude waves tested bear little similarity to the highly nonlinear, steep ocean waves encountered in typical design environmental conditions (i.e. severe storms offshore). Finally, the near-trapping phenomenon for cylinder arrays in the open ocean has not been experimentally verified.

In light of the above, this paper discusses experiments in an offshore wave basin at HR Wallingford, for which free surface elevation measurements have been made for direct comparison with diffraction theory. The simplified geometry of the experimental model (a square array of bottom-mounted cylinders) and the examination of free surface elevation allow relatively straightforward comparison with alternative theoretical and numerical data. This paper presents results for the diffraction of steep regular waves, while a companion paper (Ohl et al. 2001) examines the diffraction of irregular waves, focused wave groups, and random seas. The effects of alterations in the frequency and steepness of incident waves on the diffracted field are examined as well as the near-trapping of second-order components observed in the experiments. First-order near-trapping was not attempted due to model and facility constraints.

\section{Wave diffraction experiments}

Testing was undertaken in the Offshore Wave Basin at HR Wallingford Ltd. Both regular and irregular incident waves were produced in 104 individual tests on the multi-cylinder model. Results from these experiments are discussed in subsequent sections for regular waves and in the companion paper by Ohl et al. (2001) for irregular waves. Only a summary of the experimental methods is presented here; an extensive discussion is given by Ohl (1999).

\subsection{Description}

The three-dimensional offshore wave basin at HR Wallingford has a plan area of $25 \mathrm{~m} \times 25 \mathrm{~m}$ and operates with a working depth of $2 \mathrm{~m}$. The 80 computer controlled individual paddles at the basin's upstream end are capable of generating either regular or irregular waves with directional spreads of up to $\pm 45^{\circ}$. However, only unidirectional waves were analysed in these experiments, with the wave crests parallel to the wave paddles. At the downstream end of the basin is a beach to prevent significant reflection of wave energy. A moveable bridge spanning the basin provided access to the deck of the model structure and the wave probes. Video cameras (discussed further below) were located at the side of the wave basin both upwave and downwave of the model.

The model was placed in the centre of the wave basin, where the origin $(x=0, y=0)$ is taken for reference purposes. The $x$-axis is in the direction of incident wave propagation (the longitudinal direction), while the $y$-axis is perpendicular to the incident wave direction and parallel to the wave paddles (the transverse direction).

The diffraction model comprised a square array of four bottom-mounted vertical circular cylinders and was fabricated from standard $406 \mathrm{~mm}$ (16 in.) diameter steel pipe with thickness $6.35 \mathrm{~mm}(0.25 \mathrm{in}$.) and uniform circular cross-section (i.e. $a=203 \mathrm{~mm}$ ). Figure $1(a, b)$ provides plan view and side elevation schematics of the model. As shown, the cylinders are arranged at the corners of a square, with centre to centre distance equal to twice the cylinder diameter and a gap between cylinders of one diameter. The air gap between the mean still water level and the deck structure supporting the wave probes was selected to be $450 \mathrm{~mm}$, which was assumed to be higher than the likely diffracted free surface elevation for any of the incident waves to be considered. 
(a)

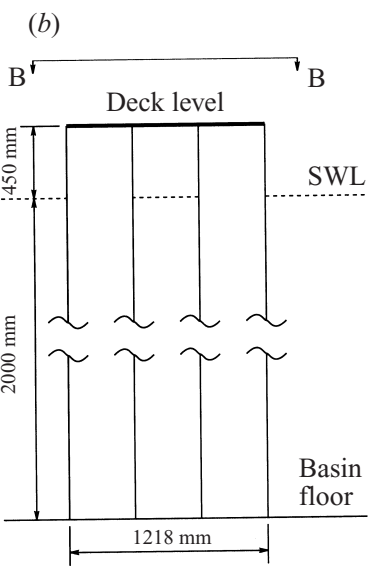

FIGURE 1. Schematic diagram of the model structure in plan view $(a)$ and side elevation $(b)$.

Due to the large mass of each cylinder (approximately $1150 \mathrm{~kg}$ ), the model was assembled inside the basin with the cylinders fixed to a square steel template on the basin floor. Two holes on the periphery of the template allowed the model to be positioned in two configurations. The first configuration, hereafter referred to as heading $0^{\circ}$, is that presented in figure 1 , with two cylinders upstream and two downstream. The second configuration, hereafter referred to as heading $45^{\circ}$, is with one cylinder upstream (centre along $y=0$ ), one downstream (centre along $y=0$ ), and two along the transverse centreline of the wave basin (centres along $x=0$ ).

A total of 13 channels was available for wave probe measurements, allowing the use of one permanently fixed wave probe (hereafter referred to as the reference probe or probe 0$)$ at $(x=-114 \mathrm{~mm}, y=3500 \mathrm{~mm})$ or $(x=-0.56 a, y=17.2 a)$ and 12 moveable wave probes in the vicinity of the model structure. A wave probe support system was mounted on the top of the cylinders. This deck structure contained a matrix of positions for the wave probes to be attached.

Video cameras were positioned to provide views from the upstream and downstream ends of the wave basin as well as between the cylinders. Signals from the cameras were fed into a split screen television, allowing both camera angles to be viewed simultaneously. This made it possible to follow the progression of a wave as it approached the model, reached the cylinders, and progressed further downstream.

\subsection{Experimental procedures}

Following model installation in the $0^{\circ}$ heading configuration and the subsequent filling of the basin, the wave probes were individually calibrated. A series of pre-calibration regular wave tests (PRWT) was then performed to identify frequencies of interest for the final test matrix as well as regions of significant upwelling and runup for wave probe placement. Following these PRWT, a test matrix was developed to examine the effects of varying both frequency and steepness for regular and irregular incident waves for both model headings. Testing then began for the $0^{\circ}$ heading case, during which the wave probes were placed in two separate configurations. Following these tests, the model was rotated through $45^{\circ}$, and the same series of incident regular and irregular waves repeated, again with two wave probe configurations. After the model tests, the deck structure supporting the wave probes was fixed in place on the basin bridge directly above the model. The basin was then emptied for model removal and subsequently refilled for a repetition of the tests, producing equivalent regular and 


\begin{tabular}{lccccccc}
\hline Test & $f[\mathrm{~Hz}]$ & $k a$ & $\lambda / h_{0}$ & $\lambda / h_{45}$ & $A_{i}[\mathrm{~m}]$ & $A[\mathrm{~m}]$ & $k A$ \\
$1 a$ & 0.8000 & 0.524 & 6.00 & 2.70 & 0.1016 & 0.0925 & 0.238 \\
$1 b$ & & & & & 0.0508 & 0.0490 & 0.126 \\
$2 a$ & 0.7838 & 0.503 & 6.25 & 2.82 & 0.1058 & 0.0985 & 0.244 \\
$2 b$ & & & & & 0.0529 & 0.0493 & 0.122 \\
$3 a$ & 0.7686 & 0.483 & 6.50 & 2.93 & 0.1101 & 0.1075 & 0.256 \\
$3 b$ & & & & & 0.0550 & 0.0571 & 0.136 \\
$4 a$ & 0.7542 & 0.465 & 6.75 & 3.04 & 0.1143 & 0.1138 & 0.261 \\
$4 b$ & & & & & 0.0572 & 0.0589 & 0.135 \\
$5 a$ & 0.7406 & 0.449 & 7.00 & 3.16 & 0.1185 & 0.1129 & 0.249 \\
$5 b$ & & & & & 0.0593 & 0.0613 & 0.135 \\
$6 a$ & 0.7277 & 0.433 & 7.25 & 3.27 & 0.1228 & 0.1150 & 0.245 \\
$6 b$ & & & & & 0.0614 & 0.0597 & 0.127
\end{tabular}

TABLE 1. Test matrix for regular waves ( $A_{i}$ is the amplitude input to the paddles, while $A$ is the measured incident amplitude).

irregular wave results in the absence of the model. (This case is referred to below as the 'empty tank'.)

From observations made during the PRWT, it was concluded that the highest frequency waves $(k a>0.4)$ showed the most significant free surface disturbances. In addition, this disturbance was related to the ratio of incident wavelength to cylinder diameter or spacing (as discussed in the Introduction). A test matrix was then constructed to analyse the effects of varying incident wave frequency based on the ratio of wavelength to cylinder diameter $(\lambda / 2 a)$. However, also of importance is the ratio of wavelength to cylinder spacing (taken as half the distance between the centres of the most upstream and most downstream cylinders), which is $\lambda / h_{0}$ and $\lambda / h_{45}$ for the $0^{\circ}$ and $45^{\circ}$ heading configurations respectively $\left(h_{0}=2 a\right.$ and $h_{45}=2 \sqrt{2} a$ ).

Six regular wave frequencies were selected for testing. For each of these frequencies, two separate values of steepness were chosen, one near to breaking (subscript $a$ ) and the other half as steep (subscript $b$ ) (target values were $\lambda / A_{a}=24, k A_{a}=0.262$ and $\lambda / A_{b}=48, k A_{b}=0.131$, respectively). Tests were also conducted in irregular waves, and these are discussed in a companion paper (Ohl et al. 2001).

The resulting test matrix for regular waves is displayed in table 1. It is important to note that all values in the test matrix related to wavenumber or wavelength have been computed through the linear dispersion equation. In addition, $A_{i}$ is the amplitude input to the wave generating equipment, while $A$ and non-dimensionalized $k A$ are the incident amplitude as measured during the experiments, and are discussed further below.

Two probe configurations were used for each model heading, designated $\mathrm{A}$ and $\mathrm{B}$ for the $0^{\circ}$ heading and $\mathrm{C}$ and $\mathrm{D}$ for the $45^{\circ}$ heading. With 12 movable wave probes and the reference probe, this provided a total of 13 wave probes in each probe configuration, with 25 distinct measurement positions for each model heading. The reference probe (probe 0) position was constant for the duration of the experiments.

The relevant probe positions are listed in table 2, in the Cartesian coordinate system introduced above, and a diagram of the approximate locations is provided in figure 2 . The probes are referenced according to probe configuration and probe number (e.g. B9 is wave probe 9 in configuration $\mathrm{B}$ for the $0^{\circ}$ heading case). For the empty tank regular wave tests, the probes were placed in configuration $\mathrm{D}$.

Between tests, a settling period of approximately 30 to 45 minutes was allotted to 
(a) Heading $0^{\circ}$

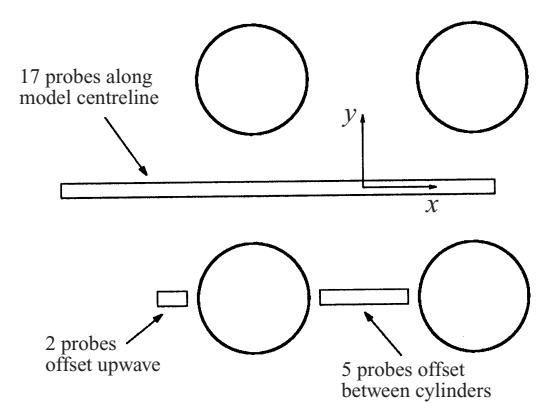

(b) Heading $45^{\circ}$
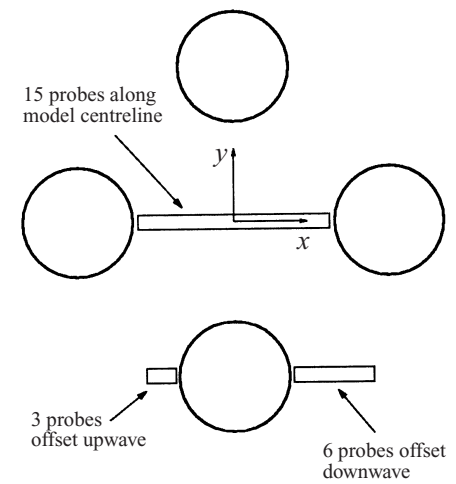

Incident wave ray

FIGURE 2. Plan view of approximate wave probe locations (see table 2 for exact positions).

\begin{tabular}{|c|c|c|c|c|c|}
\hline Probe & $x[\mathrm{~mm}]$ & $y[\mathrm{~mm}]$ & Probe & $x[\mathrm{~mm}]$ & $y[\mathrm{~mm}]$ \\
\hline A0 & -114 & 3500 & $\mathrm{C} 0$ & -114 & 3500 \\
\hline B0 & -114 & 3500 & D0 & -114 & 3500 \\
\hline B10 & -1150 & 0 & C9 & -350 & 0 \\
\hline B9 & -1050 & 0 & D6 & -300 & 0 \\
\hline B8 & -950 & 0 & C8 & -250 & 0 \\
\hline B7 & -850 & 0 & D5 & -200 & 0 \\
\hline B6 & -750 & 0 & C7 & -150 & 0 \\
\hline B5 & -650 & 0 & D4 & -100 & 0 \\
\hline B4 & -550 & 0 & C6 & -50 & 0 \\
\hline B3 & -450 & 0 & C5 & 0 & 0 \\
\hline A12 & -350 & 0 & $\mathrm{C} 4$ & 50 & 0 \\
\hline A11 & -250 & 0 & D3 & 100 & 0 \\
\hline A10 & -150 & 0 & $\mathrm{C} 3$ & 150 & 0 \\
\hline A9 & -50 & 0 & D2 & 200 & 0 \\
\hline A 8 & 50 & 0 & $\mathrm{C} 2$ & 250 & 0 \\
\hline A7 & 150 & 0 & D1 & 300 & 0 \\
\hline A6 & 250 & 0 & $\mathrm{C} 1$ & 350 & 0 \\
\hline A5 & 350 & 0 & C12 & -325 & -575 \\
\hline A4 & 450 & 0 & C11 & -275 & -575 \\
\hline B12 & -765 & -407 & C10 & -225 & -575 \\
\hline B11 & -665 & -407 & D12 & 220 & -575 \\
\hline B2 & -150 & -407 & D11 & 320 & -575 \\
\hline B1 & -50 & -407 & D10 & 370 & -575 \\
\hline A3 & 50 & -407 & D9 & 420 & -575 \\
\hline A2 & 100 & -407 & D8 & 470 & -575 \\
\hline A1 & 150 & -407 & D7 & 520 & -575 \\
\hline
\end{tabular}

TABLE 2. Wave probe positions for $0^{\circ}(\mathrm{A}$ and $\mathrm{B})$ and $45^{\circ}(\mathrm{C}$ and $\mathrm{D})$ headings.

allow for the damping of any standing waves in the basin. This settling time also allowed the wave probes to be zeroed prior to the start of each test. Data acquisition began when the wave paddles were first activated and continued until input to the paddles ceased, a total duration of 100 wave periods (i.e. $125 \mathrm{~s}$ of data for the highest regular wave frequency of $f=0.8000 \mathrm{~Hz}, T=1.25 \mathrm{~s}$ ). 
Measurements of free surface elevation by the wave probes were recorded at a rate of 40 measurements per wave period, corresponding to a sampling rate of $40 f$ (i.e. sampling at $32 \mathrm{~Hz}$ for $f=0.8000 \mathrm{~Hz}$ ).

\subsection{Potential errors and limitations}

Possible errors in the data could result from either the presence of undesirable disturbances in the wave basin or motion of the wave probes. Undesirable disturbances could be caused by residual energy from previous tests due to insufficient settling time or the slow accumulation of reflected energy from incomplete damping by the beach. Any motion of the wave probes could be due to direct stimulation by incident waves or indirectly through motion of the model and deck structure caused by wave forces. However, none of the above sources of error were observable during the testing, and the data collected are believed to be of high quality.

Possible limitations should be noted with regard to the measurement of the free surface during particularly violent events. The resistance wave probes utilized in the experiments depend on a continuous free surface between the rods in order to register an elevation reading. Given the spacing between the rods $(12 \mathrm{~mm}$ distance centre to centre), it is reasonable to suggest that any spray or foam generated for waves of high steepness would not have been recorded. In addition, the wave probes and associated data collection equipment are capable of registering only one distinct elevation per time interval, thus assuming that the free surface is single-valued (i.e. non-overturning).

\section{Results of regular wave diffraction experiments}

The data analysis from the multi-cylinder diffraction experiments is now described in detail, and the results from the regular wave tests are discussed with reference to linear and second-order diffraction theory. While regular waves were tested for six frequencies as presented in the test matrix of table 1, results are presented here for only the highest and lowest test frequencies, with some discussion of the neartrapping phenomenon observed at an intermediate frequency. Complete results at all test frequencies are presented by Ohl (1999).

In the following discussion, cylinders are distinguished as upwave and downwave in both heading cases and as offset for the centreline cylinders of the $45^{\circ}$ heading cases. The upwave and downwave cylinder edges are referred to as the front and rear faces, respectively. The terms inboard and outboard are used to describe direction toward and away from the centre of the model, respectively, and longitudinal and transverse describe general orientation parallel and perpendicular to the incident wave ray, respectively.

\subsection{Video observations}

The camera angles chosen are detailed in figures $3(a)$ and $3(b)$, and are discussed further below. Videos of the regular wave tests are used primarily for qualitative analysis to provide a general description of the wave scattering processes. Such a general description is presented first, with subsequent presentation of comparable theoretical results in the form of contour plots. The qualitative video observations are then compared with the theoretical contour plots.

Discussion of video observations is divided according to wave heading. The overall observed process is first discussed based upon the lower steepness cases, as these involve less spray and foam and are thereby easier to characterize. Simplistic plan 

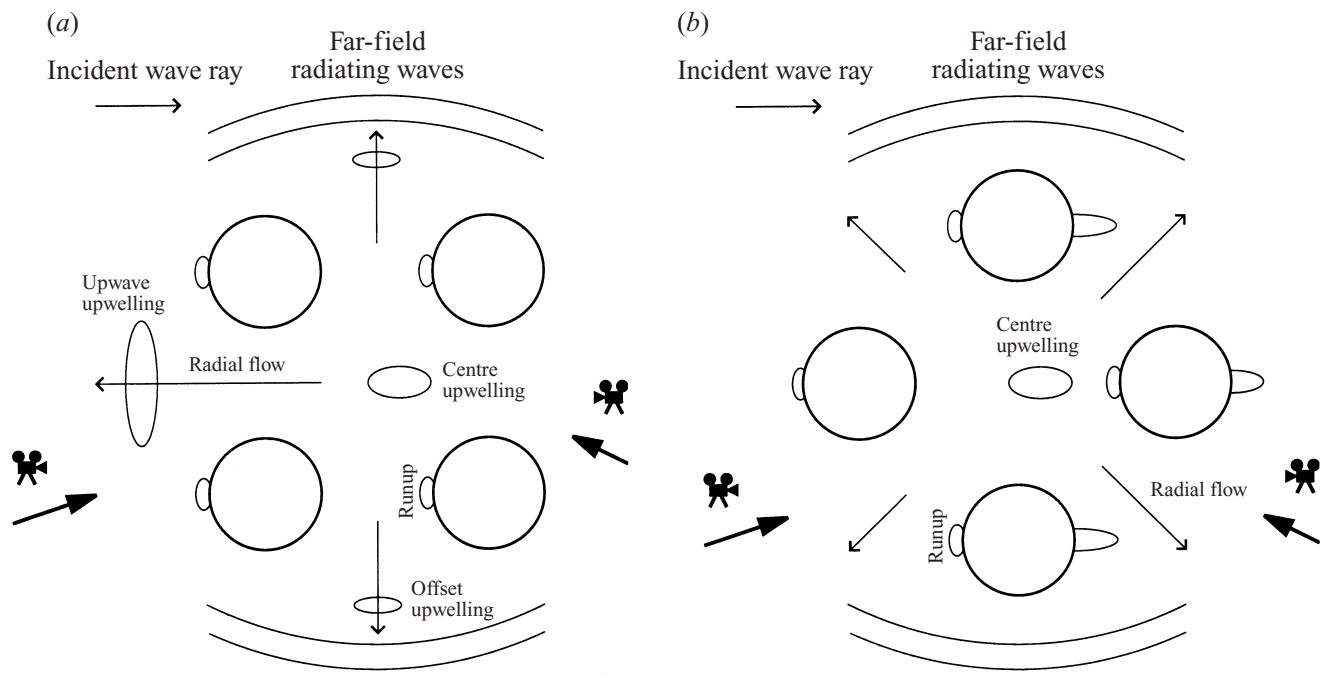

FIGURE 3. Diagram of general video observations with camera angles indicated: (a) $\beta=0^{\circ}$, (b) $\beta=45^{\circ}$.

drawings in figure 3 show these general processes. Cylinder positions are clearly defined with thick line circles, arrows indicate the direction of the incident wave and any scattered waves, arcs show the presence of radiating waves, and ellipses and partial ellipses are at the locations of upwelling and runup on the cylinders.

This overview of the video observations is then followed by a description of the time evolution of a wave approaching the model. Finally, differences in the behaviour due to changes in wavelength and the higher steepness cases are discussed.

\subsubsection{Heading $0^{\circ}$}

During the $0^{\circ}$ heading tests, the behaviour of the free surface is primarily characterized by a large upwelling at the centre of the model, beneath the deck structure. This event causes the radial flow of liquid from the centre of the model. As illustrated in figure 3(a), this upwelling and subsequent radial flow generates far-field radiating waves as well as large sites of increased wave amplitude upwave and to either side of the model.

As a wave crest approaches the model, its amplitude visibly increases just upwave of the model as it meets a stream of fluid being expelled upwave from the model centre. The amplitude again increases at the point of runup at the upwave cylinder front face. Subsequently, as the wave proceeds beneath the deck structure, a massive upsurge is observed at the model centre. This central upsurge is accompanied by upwelling to either side of the model, approximately 2.5 diameters from the model centre. As the central mound of water collapses, liquid moves outward radially from beneath the structure. This results in a high-frequency radiating wave at two or three times the incident frequency; and, as previously mentioned, a stream is forced upwave to interact with the next incoming wave.

More specifically, as the wavelength of the incident wave increases (decreasing frequency), the two primary locations of upwelling (at the model centre and just upwave of the model) move progressively upwave. As incident wave steepness increases, the overall observed processes are similar, but there is significant enhancement in the violence of the aforementioned upwelling and radiation events. There is a large 
amount of foam and spray generated by the central upwelling at higher steepness, but the free surface does not contact the lower deck structure, and the spray generated by upwelling and runup does not extend appreciably above deck level. In addition, at higher steepness, the liquid expelled upwave appears to breakup as it meets the incoming incident wave.

\subsubsection{Heading $45^{\circ}$}

Again, the most visible event during the $45^{\circ}$ heading tests is massive upwelling, and possible formation of a clapotis (a large reflected wave resembling a standing wave), at the model centre. This upwelling generates radiating waves, but liquid expulsion from the centre does not appear as dramatic as that observed during the $0^{\circ}$ heading tests described above. Figure $3(b)$ indicates the centre upwelling as well as the principal regions of runup, observed at the front and rear faces of the offset and downwave cylinders.

Upwave of the model, the incident wave remains undisturbed until the crest reaches the upwave cylinder and runup amplification occurs. After the wave crest passes the upwave cylinder, runup amplification occurs again at the front faces of the offset cylinders. Again, large upwelling occurs as the wave proceeds beneath the deck structure and appears to be the direct source of far-field waves radiating away from the model at two or three times the incident wave frequency. This amplified wave progresses towards the downwave cylinder, where significant runup occurs, while expelling fluid between the offset and downwave cylinders. This stream of fluid causes large runup both at the rear faces of the offset cylinders and, as the incident wave passes the downwave cylinder, at the rear face of the downwave cylinder.

The timing of observed events at the model centre varies somewhat with the incident wave frequency. At the highest two frequencies, $f=0.8000$ and $0.7838 \mathrm{~Hz}$, runup at the front face of the downwave cylinder occurs shortly before runup at the rear faces of the offset cylinders. With increasing wavelength, runup at the offset cylinders begins to precede that at the downwave cylinder. As above for the $0^{\circ}$ heading, the location of the maximum centre upwelling moves steadily upwave with increasing wavelength (decreasing frequency). Again, at higher wave steepness, the centre upwelling and runup at the cylinders produces large amounts of spray and foam, but the free surface does not make contact with the deck structure, and the spray does not extend appreciably or consistently above the deck (except in one case discussed further below).

In addition, with increasing wavelength the location of maximum runup at the rear face of the offset cylinder shifts steadily further away from the model centre. This shift is more noticeable in the higher steepness cases, in which this runup occurs directly downwave at $f=0.8000 \mathrm{~Hz}$ but swings steadily outboard to approximately $45^{\circ}$ at $f=0.7227 \mathrm{~Hz}$.

Finally, there is a notable increase in the overall violence of the process, to include the centre upwelling and runup at the offset cylinder's rear face, at $f=0.7542 \mathrm{~Hz}$. For the higher steepness case at this frequency, spray generated by front face runup on the downwave cylinder rises to approximately $200 \mathrm{~mm}$ above the deck (the underside of which, it may be recalled, is $450 \mathrm{~mm}$ above the still water level).

\subsection{Contour plots of linear free surface amplitude}

As a means of comparison with the video data, contour plots of the local linear free surface amplitudes were produced through the analytical method of Linton \& Evans (1990). These contour plots are presented here in figure 4(a) for heading $0^{\circ}$ and 
(a)

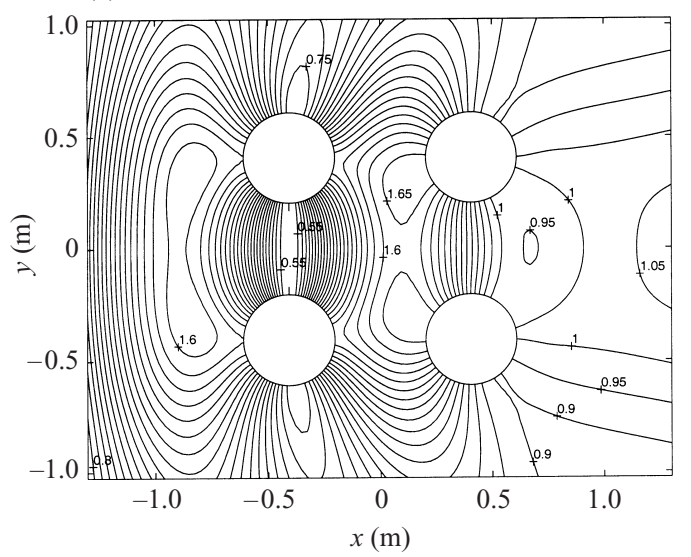

(b)

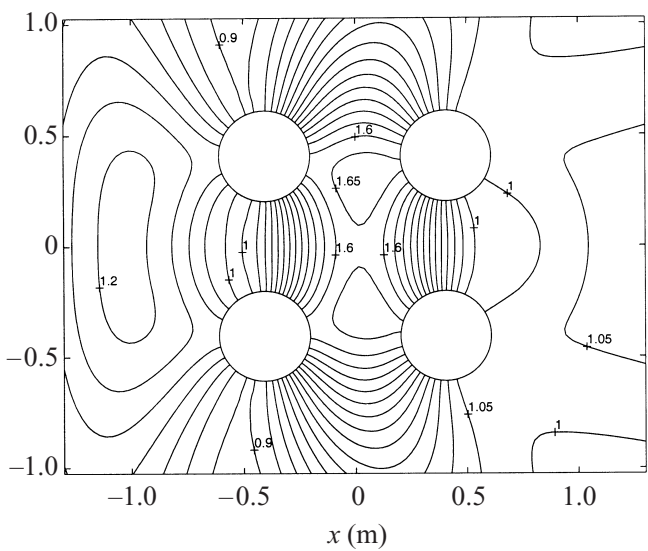

Figure 4. Linear free surface amplitude; $\beta=0^{\circ}:(a) f=0.8000 \mathrm{~Hz},(b) f=0.7277 \mathrm{~Hz}$.

(a)

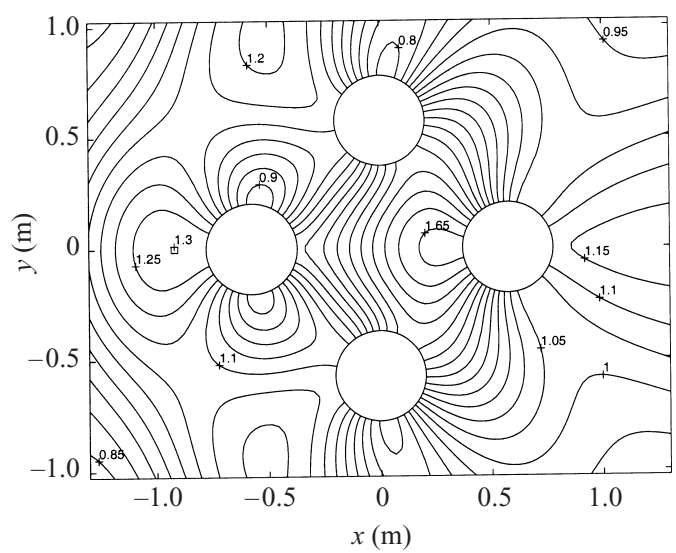

(b)

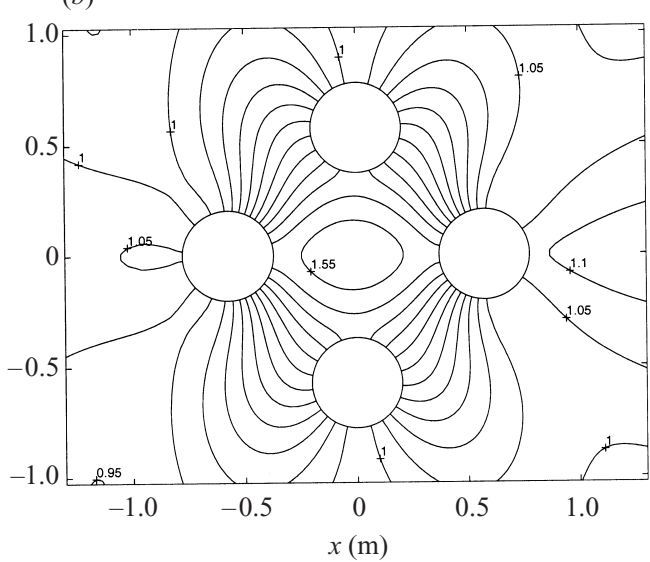

FIGURE 5. Linear free surface amplitude; $\beta=45^{\circ}:(a) f=0.8000 \mathrm{~Hz},(b) f=0.7277 \mathrm{~Hz}$.

figure $4(b)$ for heading $45^{\circ}$. Solid circles denote the cylinder positions. Key contours are labelled with magnitude in terms of the amplification modifying the incident wave. For example, a contour labelled 1 indicates no modification to the incident wave amplitude along that line, while a value of 1.5 corresponds to a $50 \%$ increase in amplitude. As before, discussion of these plots is separated according to heading.

\subsubsection{Heading $0^{\circ}$}

The principal features of the plots are broad regions of upwelling upwave of the model and at the model centre. Between these peaks of upwelling, a region of diminished wave amplitude runs transversely between the two upwave cylinders, while small lobes of decreased amplitude exist outboard of the upwave cylinders. Significant runup is indicated at the front and rear faces of the upwave cylinders and at the front faces of the downwave cylinders. Any modifications to the incident wave amplitude diminish greatly with increased distance away from the model, most particularly downwave, where very little modification is observed past the downwave cylinders. 
With increasing wavelength and decreasing frequency, the upwave upwelling broadens longitudinally but decreases in amplitude (by approximately $25 \%$ ). In contrast, the centre upwelling remains reasonably constant at approximately 1.65 times the incident wave amplitude. Both upwelling regions shift steadily upwave with increasing wavelength, while the diminished amplitude region between the upwave cylinders becomes less pronounced. Indeed, at $f=0.7277 \mathrm{~Hz}$ in figure $4(b)$, virtually no modification of the incident wave amplitude occurs between the upwave cylinders.

\subsubsection{Heading $45^{\circ}$}

The contour plots feature a significant region of upwelling and runup throughout the centre of the model, which includes runup at all inboard cylinder edges. In addition, some runup occurs at the front face of the upwave cylinder and at the rear face of the downwave cylinder. Notably, no major areas of diminished wave amplitude are observed apart from small lobes at the outboard edges of the offset cylinders and the transverse edges of the upwave cylinder, which are present only at the highest frequencies (such as $f=0.8000 \mathrm{~Hz}$ in figure $5(a)$ ).

At the higher frequencies, a small region of upwelling upwave of the model meets the runup at the front face of the upwave cylinder. In addition, the upwave cylinder is flanked by upwelling approximately 2.5 diameters from the cylinder centre, and runup is indicated at both the front and rear faces of the offset cylinder. With decreasing frequency and increasing wavelength, the upwelling and runup at the upwave cylinders diminish considerably in height and the flanking regions disappear entirely. Again, the strength of the centre upwelling remains reasonably constant, decreasing from approximately 1.65 to 1.55 times the incident wave amplitude with increasing wavelength. As in the $0^{\circ}$ heading case described above, the position of maximum upwelling at the model centre shifts upwave with increasing wavelength. Finally, from an aesthetic perspective, the contour plots become fairly symmetric at the lowest test frequencies (such as $f=0.7277 \mathrm{~Hz}$ in figure $5(b)$ ). This symmetry is unique to these frequencies and is not present in contour plots at lower frequencies outside the test range (not presented here).

\subsection{Comparison of video observations with contour plots}

For the most part, the global features that can be observed in the videos are very similar to those found in the contour plots. For the $0^{\circ}$ heading cases, the extreme upwelling observed upstream and near the model centre agree well with the firstorder theoretical predictions. This is also true for the runup at the front face of both upwave and downwave cylinders. However, the offset upwellings observed some 2.5 diameters to either side of the model are not predicted by the theory. It is possible that this offset upwelling is generated by a second- or third-order event related to energy radiation from the structure in the near field. Regions of diminished wave amplitude in the contour plots, such as the lobes outboard of the upwave cylinders and the region in between these two cylinders, are difficult to identify in the video data. Similarly, at $45^{\circ}$, the locations of significant upwelling and runup at the model centre are predicted well by the theory. In addition, the runup sites at the upwave cylinder's front face and the downwave cylinder's rear face are as in the contour plots. Finally, the upwave shift of the global features in the contour plots of both cases is consistent with the video observations. 


\subsection{Data manipulation}

Free surface elevation data $\eta(t)$ from the regular wave tests with the model present were analysed to provide first-, second- and third-order free surface amplitudes at each wave probe position. These amplitudes were then compared to incident wave amplitudes as computed from empty tank tests.

\subsubsection{Data record truncation}

The initial data records from the regular wave tests begin at the start of the wavemaker motion and end approximately 2 minutes later. As such, they include an initial timespan during which full wave height had not been achieved as well as an end period during which reflected waves from the tank boundary opposite the wavemaker had arrived at the model. The usable middle section of the data was then identified by analysing complete time histories to determine the point after which waves had stabilized near their intended height and, subsequently, the point after which some reflection or other interference was visibly altering this sustained height.

This data record truncation is illustrated in figure $6(a)$, which contains the majority of three data records, and figure $6(b)$, which provides more detail of the isolated segments. This figure plots free surface elevation versus time from wave probe locations near the tank centre from model tests at $0^{\circ}$ and $45^{\circ}$ headings as well as the empty tank tests, for the lower-steepness case at $f=0.7542 \mathrm{~Hz}$. It is clear from figures $6(a)$ and $6(b)$ that, by the start of the truncated data section, waves at the model centre are approaching a steady-state condition. While it is more difficult to discern the point at which reflections in the wave basin have disturbed this steady-state condition, it is reasonable to suggest that this occurs after the truncated section.

\subsubsection{Frequency analysis, amplitude computation, set-up and set-down computation}

Fast Fourier transforms (FFTs) of the data were used to produce a frequency domain analysis for each wave probe. Spectral peaks at the incident frequency, double frequency and triple frequency (frequencies equivalent to $f=f_{i}, 2 f_{i}$ and $3 f_{i}$, where $f_{i}$ is the incident wave frequency) were then separated by removing all spectral components other than those at or near the peak (within $0.25 f_{i}$ above or below). Components at these frequencies will hereafter be referred to as first-, secondand third-order harmonics for the single, double and triple frequencies, respectively. Inverse FFTs of the modified spectra produced time series plots of the separate components, from which a mean amplitude could be computed. In addition, the magnitude of the set up and set down was calculated from the mean value of the time histories.

\subsection{Data presentation and observations}

To best represent the data, computed experimental values were plotted versus the wave probe location. In the descriptions of observed phenomena, a distinction is made between centreline plots (along the tank and model longitudinal centreline at $y=0$ ) and offset plots (to the side of the model at $y=-2 a$ or $y \approx-2.83 a$ ). For reference, the transverse centreline runs parallel to the wave paddles at $x=0$.

\subsection{Definition of incident wave amplitude}

In order to properly non-dimensionalize the data, the incident wave amplitude was taken to be the amplitude of the linear frequency component of the incident wave as measured during the empty tank tests. Use of the empty tank tests to define the 
Wave diffraction. Part 1. Regular waves

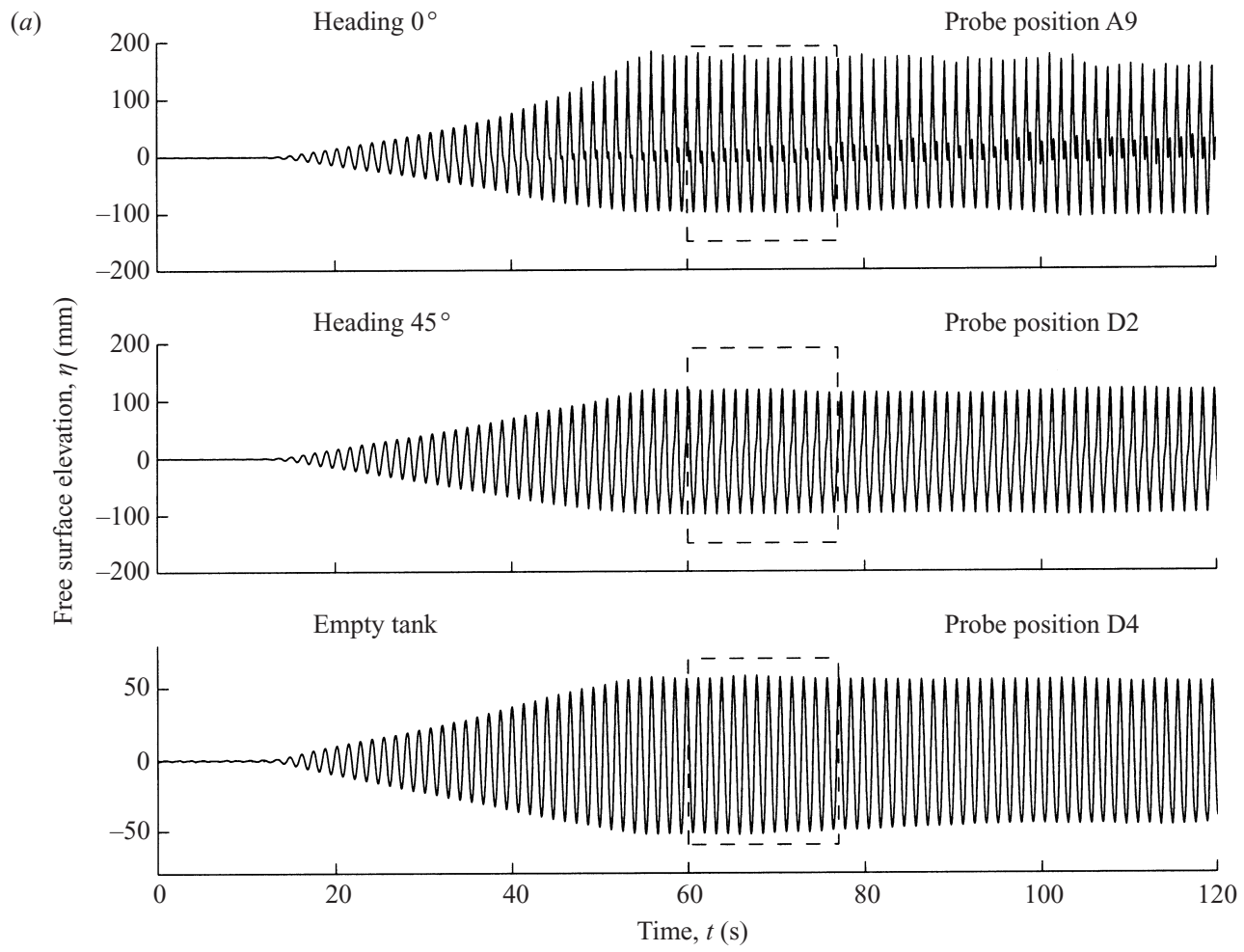

(b)

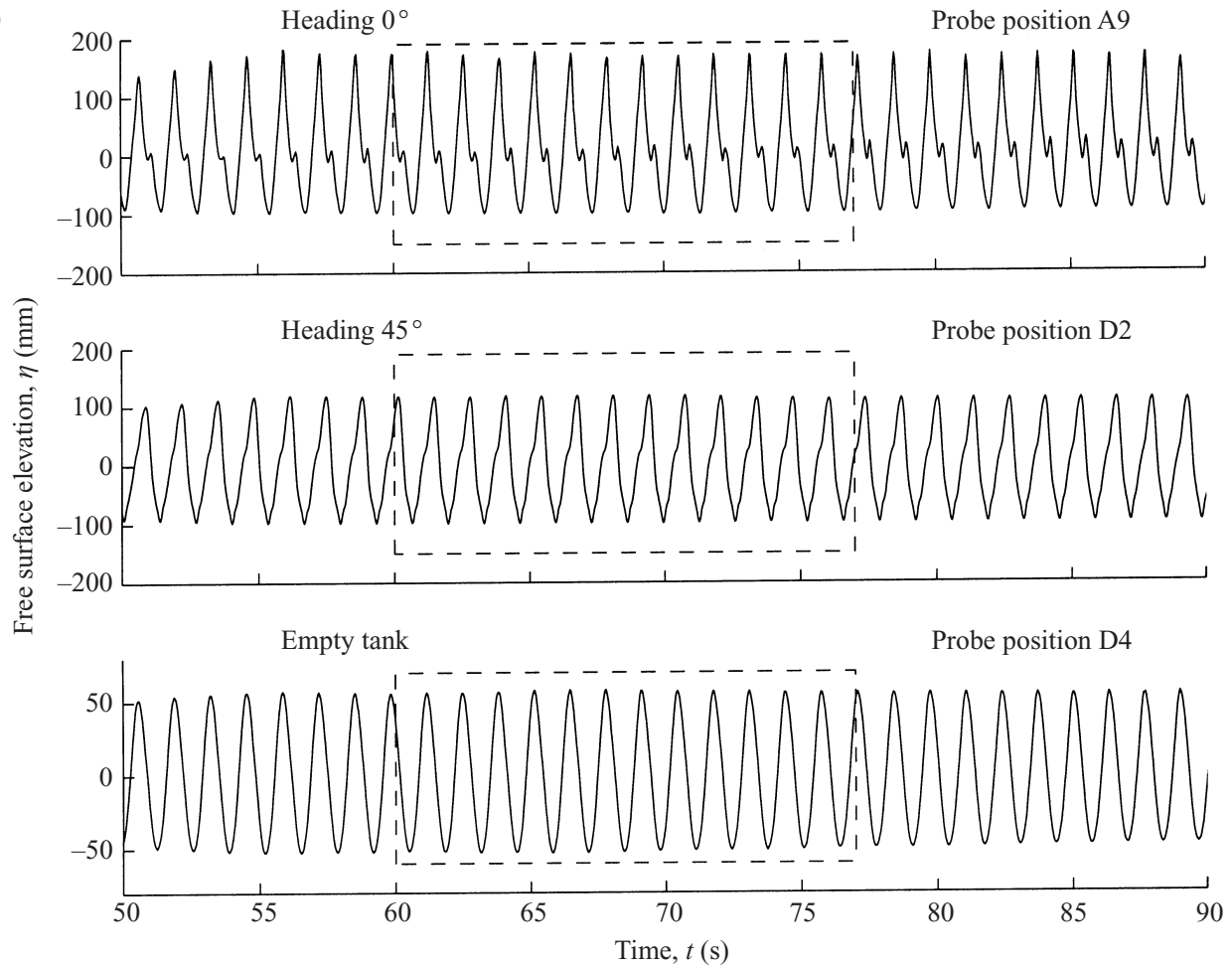

FigURE 6. Free surface elevation vs. time for $(a)$ the entire data record and $(b)$ partial data record; $\beta=0^{\circ}, 45^{\circ}$ and the empty tank case, $f=0.7542 \mathrm{~Hz}$, lower-steepness case; from locations near the tank centre. The data section used is shown in the dashed box. 
undisturbed incident wave ensured the removal of any far-field disturbances that may have been generated by the presence of the model.

As discussed previously, table 1 gives the incident wave amplitude, $A$, and the non-dimensionalized values $k A$ for each test frequency. It is apparent from the table that, although the waves used in the experiments were not calibrated prior to testing, the amplitudes observed are reasonably close to the target steepness.

\subsection{Amplitude variation plots and linear theory comparison}

For each test frequency, amplitude versus position were plotted for the properly non-dimensionalized first-, second- and third-order components. The results for the highest and lowest test frequencies are presented in figures 7 and 8 for heading $0^{\circ}$ and figures 9 and 10 for heading $45^{\circ}$. Part $(a)$ of each figure presents first-, second- and third-order amplitudes (from top to bottom) as computed along the centreline of the tank $(y=0)$, while part $(b)$ presents the offset amplitude computations $(y=-2 a$ or $-2.83 a)$. The ' $x$ ' and ' + ' symbols are used for the higher- and lower-amplitude cases, respectively, and straight lines are drawn to denote the cylinder positions.

Linear diffraction theory was used to compute a theoretical prediction corresponding to the experimental results. This comparison is presented as the solid line in the upper two plots of figures 7 to 10 .

Direct observations which can be made from figures 7-10 are grouped herein according to heading and order, beginning with the $0^{\circ}$ heading, first-order plots and proceeding to the $45^{\circ}$ heading, third-order plots. A more general discussion of the overall effects observed, and possible reasons for some counter-intuitive features in the results, are given in $\S 4$. Where possible, observed trends in the higher-order plots are described with reference to the corresponding first order plot. Regions or point locations of amplification are referred to as peaks, while regions of diminished amplitude are referred to as troughs. It should be kept in mind that use of these words bears no resemblance to the crests and troughs of waves as measured in the time or spatial domains.

\subsubsection{Heading $0^{\circ}$ : first-order observations}

With some notable exceptions, the experimental and theoretical results at first order are in close agreement. There is also little difference between results at lower and higher amplitudes.

For the centreline plots, the overall trends in both theory and experiment indicate two large peaks, one approximately two cylinder diameters $(4 a)$ upwave of the model centre and the other near the model centre. At longer wavelengths (lower frequencies), both of these peaks shift slightly further forward and the upwave peak loses strength in relation to the central peak.

Whereas the match between experiment and theory is close, there are discernible differences in the location of the minimum amplitude, the magnitude of the upwave peak, and the profile of the offset plots. In all cases, the measured minimum is approximately $5 \mathrm{~cm}$ downwave of the theoretical prediction. In addition, at higher amplitude the height of the upwave peak is significantly greater than the theory and the lower-amplitude results for four of the six test frequencies, for example at $f=0.7277 \mathrm{~Hz}$ in figure 8 . In contrast, the lower-amplitude upwave peak is the greatest only for $f=0.8000 \mathrm{~Hz}$, the highest test frequency, as shown in figure 7 . In one extreme example (at $f=0.7838 \mathrm{~Hz}$ ), the high-amplitude result is approximately $25 \%$ greater than the low-amplitude result and $50 \%$ greater than the prediction by first-order theory. 
(a)
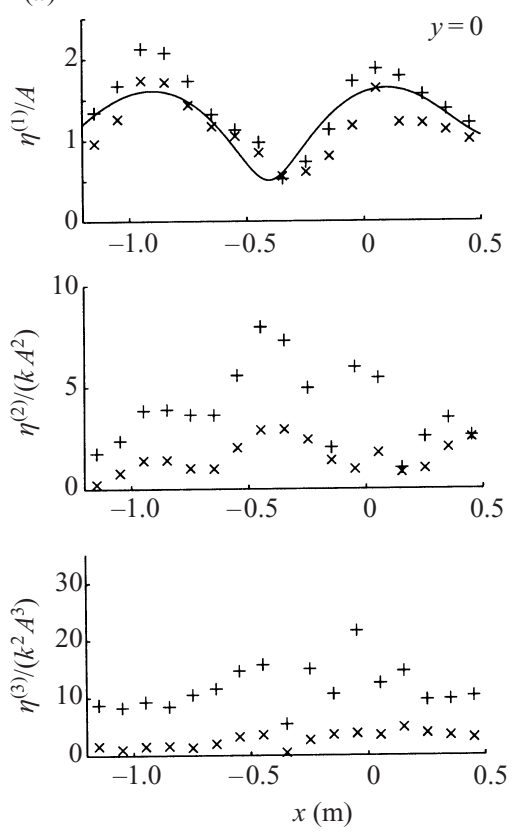

(b) $\quad \times, A=0.092452 \mathrm{~m} ;+, A=0.048959 \mathrm{~m}$
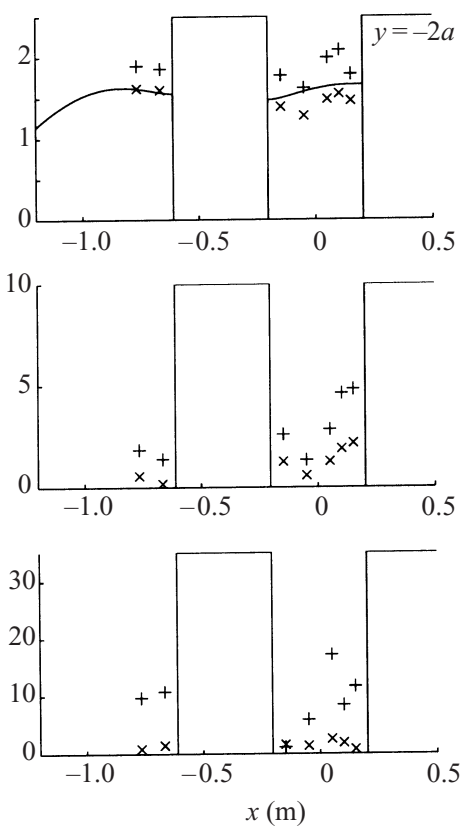

FIgURE 7. Amplitude versus position; $\beta=0^{\circ}, f=0.8000 \mathrm{~Hz}$.

(a)
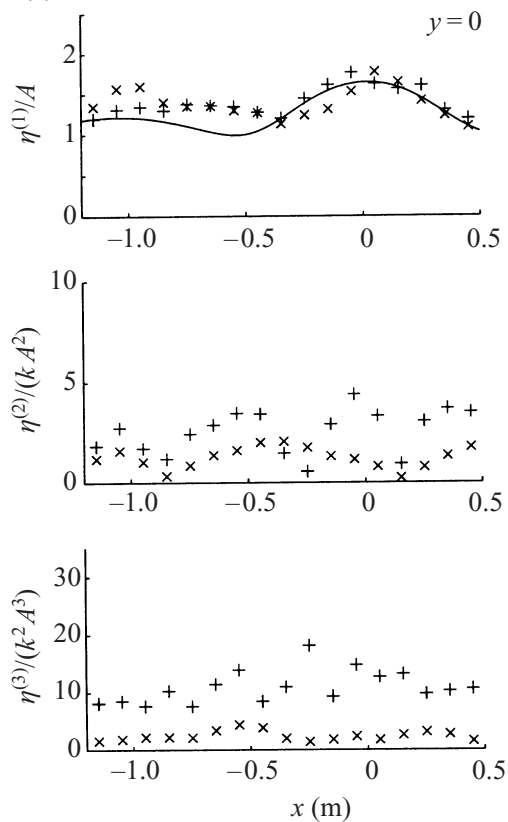

(b) $\times, A=0.11501 \mathrm{~m} ;+, A=0.059746 \mathrm{~m}$
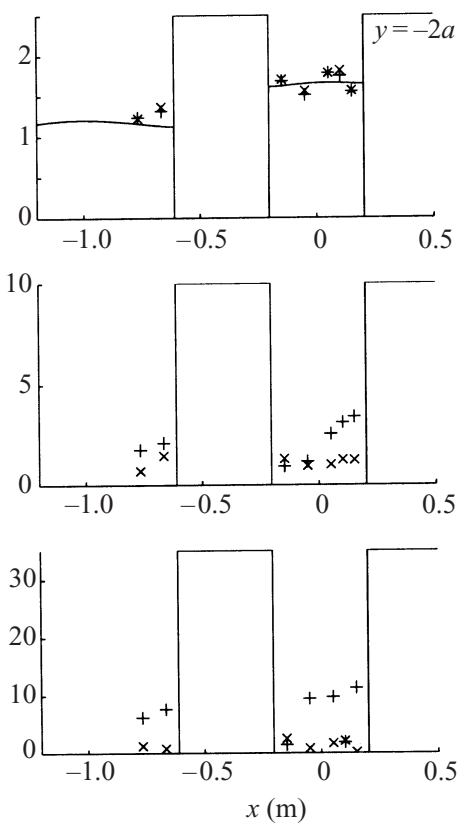

FIGURE 8. Amplitude versus position; $\beta=0^{\circ}, f=0.7277 \mathrm{~Hz}$. 
(a)
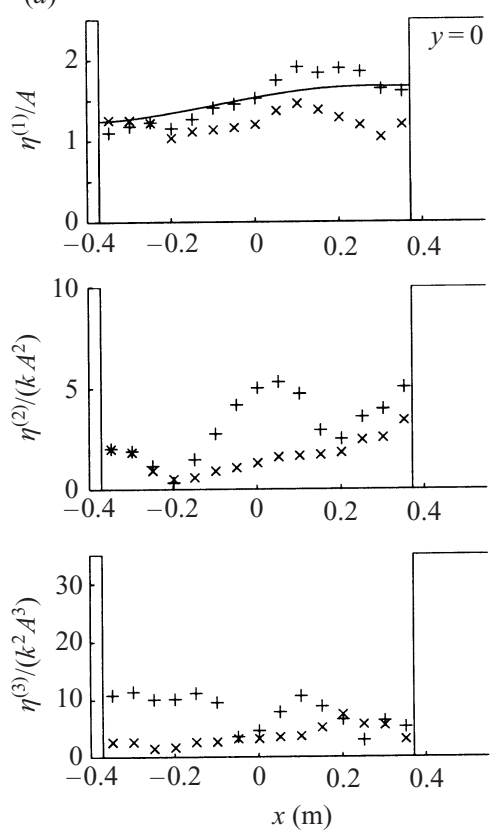

(b) $\quad \times, A=0.092452 \mathrm{~m} ;+, A=0.048959 \mathrm{~m}$
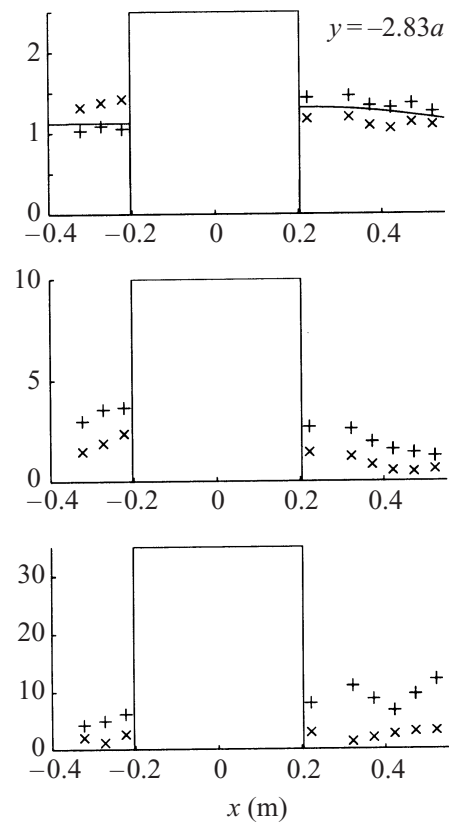

FIgUre 9. Amplitude versus position; $\beta=45^{\circ}, f=0.8000 \mathrm{~Hz}$.

(a)
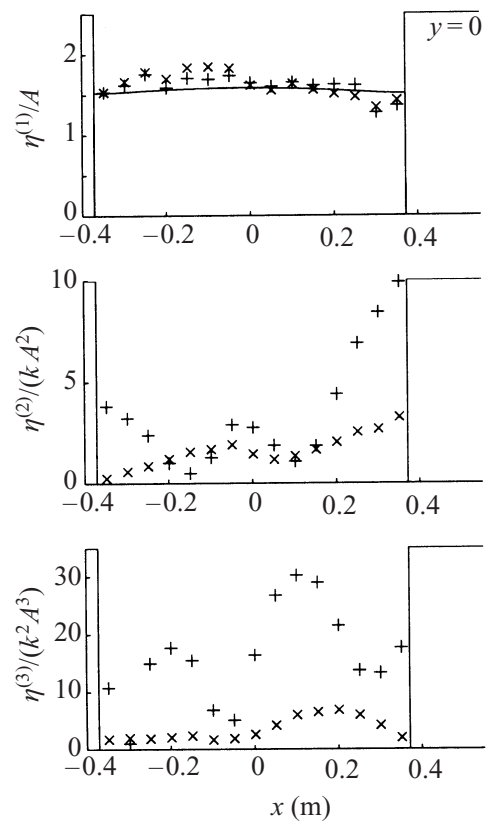

(b) $\quad \times, A=0.11501 \mathrm{~m} ;+, A=0.059746 \mathrm{~m}$
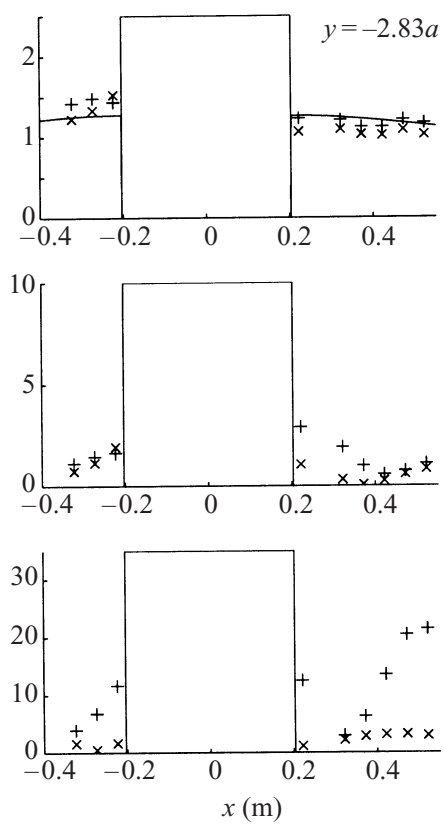

Figure 10. Amplitude versus position; $\beta=45^{\circ}, f=0.7277 \mathrm{~Hz}$. 
Finally, the offset plots yield significant differences between the two experimental profiles as well as between theory and experiment. Differences between the values at higher and lower amplitudes are varying and hard to characterize. Both experimental profiles upwave of the model seem to correspond roughly to the theoretical prediction. However, between the cylinders, the theoretical profile is characterized by a positive slope from the rear face of the upwave cylinder to the front face of the downwave cylinder, while the experimental results exhibit more complex behaviour. For all frequencies and both amplitudes, the experimental results show a peak at the rear face, followed by a trough near the transverse centreline $(x \approx 0)$, a further peak nearer to the downwave cylinder, and, finally, a trough at the front face of the downwave cylinder. Although the resolution of this profile is inadequate due to the wave probe spacing, it is apparent that the peak near the downwave cylinder shifts upstream as the wavelength increases (decreasing frequency).

\subsubsection{Heading $0^{\circ}$ : second- and third-order observations}

At second and third order, the lower-amplitude plots show significantly higher non-dimensional peaks. However, it is important to note that both the higher- and lower-amplitude plots follow similar trends in the location of peaks and troughs. Also, the maximum height of the second-order peaks is significantly greater in the small-wavelength (high-frequency) cases, while the third-order peaks are of similar maximum height at all frequencies.

In general, the second-order profiles contain peaks corresponding to peaks and troughs in the first-order profiles; and second-order troughs occur at maximum slopes in the first-order profiles. In contrast, third-order peaks occur at locations of peaks and maximum slopes in the first-order profiles, while third-order troughs occur at the first-order troughs and any gradual slopes or crest transition points (i.e. where a peak is transitioning to a slope, or vice versa). However, the largest third-order peaks occur at the first-order maximum slopes, with less significant third-order peaks at the first-order peaks. This behaviour can be clearly observed in figure 8, where the largest third-order peak occurs at $x \approx-0.25 \mathrm{~m}$, near the midpoint of the firstorder profile's transition from a trough to a peak. As the locations of second- and third-order peaks are dependent upon the first-order profile, the peaks shift steadily upwave with increasing wavelength, as described above.

While the second- and third-order offset plots are more difficult to characterize in terms of any correspondence with the first-order profiles, their behaviour is similar at most test frequencies, with a notable exception being the second-order runup. At the upwave cylinder's front face, the second-order profile shows a gradual decrease approaching the cylinder for three frequencies $(f=0.8000,0.7838$ and $0.7542 \mathrm{~Hz})$, but an increase is observed for the others $(f=0.7686,0.7406$ and $0.7277 \mathrm{~Hz})$. This seems to indicate some transition of response for the second-order runup near these frequencies $(f=0.7686$ and $0.7542 \mathrm{~Hz})$. In contrast, the lower-amplitude third-order profiles consistently show a gradual increase approaching the upwave cylinder's front face at all test frequencies, while the higher-amplitude third-order profiles exhibit very low non-dimensional values.

In the offset plots between the cylinders, the second-order amplitude profile peaks at the rear face of the upwave cylinder, dips to a trough near the transverse centreline $(x \approx 0)$, then increases to peak again at the front face of the downwave cylinder. At third order, the profile exhibits a trough at the rear face of the upwave cylinder, rises to a peak near the transverse centreline, falls again into a trough, and finally peaks at the front face of the downwave cylinder. It is apparent that the third order transverse 
centreline peak either shifts upwave or broadens as the wavelength increases and frequency decreases. However, it is impossible to distinguish which of these two is occurring because of the wave probe spacing and, in particular, the lack of a probe at $x \approx 0$, where the third-order transverse centreline peak might be expected at the lowest test frequencies (such as $f=0.7277 \mathrm{~Hz}$ in figure 8). Finally, it is important to note that the higher-amplitude plots show extremely low values in the offset cases, even in comparison with the corresponding centreline plots.

\subsubsection{Heading $45^{\circ}$ : first-order observations}

Again, with some exceptions, similar experimental and theoretical results are obtained at first order, though there is more difference between the lower- and higheramplitude results in figure 9. It is important to note that the magnitude of the diffracted first-order amplitude never drops below that of the incident wave amplitude (that is, none of the plotted first-order values are lower than unity), unlike the $0^{\circ}$ heading cases.

For the centreline plots, the overall trends in both theoretical and experimental data indicate a general area of upwelling at the model centre between the upwave and downwave cylinders, with a slight peak in the vicinity of the downwave cylinder. As the wavelength increases (decreasing frequency), the amplitude profile becomes more uniform, with the peak shifting slightly upwave and virtually disappearing at the longest wavelengths. This upwave shift of the global feature is similar, though less extreme, to that observed in the $0^{\circ}$ heading case.

The trends of the higher- and lower-steepness cases are virtually identical; but the lower steepness yields a slightly higher upwelling at all the frequencies apart from the lowest ( $f=0.7277 \mathrm{~Hz}$ in figure 10), at which the higher-steepness result exceeds the lower very slightly at the peak. The theoretical results also appear to be a better match for the lower-steepness data at all the frequencies. While the overall magnitude of the centre upwelling is well matched by the theory, more subtle features are not exactly duplicated. This is particularly noticeable at the three highest frequencies, for example at $f=0.8000 \mathrm{~Hz}$ in figure 9 . The experimental values rise slightly from the rear face of the upwave cylinder to a small peak, followed by a slight dip at $x \approx-0.2 \mathrm{~m}$. Subsequently, there is a steady rise in amplitude to a more extreme peak upwave of the downwave cylinder $(x \approx 0.2 \mathrm{~m})$, followed by a more dramatic decline and final increase in amplitude very near the front face of the downwave cylinder.

Finally, the offset plots yield only small differences between the theoretical and experimental results. The general trend is a slight increase in upwelling approaching the front face of the offset cylinder, followed by a slight decrease downwave of the rear face. The experimental results indicate a somewhat steeper increase approaching the front face, and a slight peak appears to exist just downwave of the offset cylinder (at $x \approx 0.45 \mathrm{~m}$ ) in certain cases. While there are some slight differences in the diffracted amplitudes at the two steepness values, these variations are not consistent nor are they frequency dependent.

\subsubsection{Heading $45^{\circ}$ : second- and third-order observations}

As in the $0^{\circ}$ heading cases above, the lower-amplitude plots show significantly higher peaks. But, unlike the $0^{\circ}$ heading cases, the trends in the location of peaks and troughs are not entirely consistent at second order and are significantly different at third order. In terms of magnitude, the height of the second-order centre peak is reasonably consistent at all frequencies, while the runup at the downwave cylinder's front face is significantly greater at longer wavelength and lower frequency. Similarly, 
the heights of the third-order peaks are greatly increased at longer wavelength. In addition, the experimental results vary greatly between the two steepness values, and the lower-steepness cases will be treated first and more thoroughly as their behaviour is more consistent with that previously observed in the $0^{\circ}$ heading cases.

Again, for the centreline plots at the lower steepness, the third-order profiles exhibit more rapid variation than those at second order. Ignoring the aforementioned firstorder profile complexities and idealizing the profiles as peaking in the model centre and sloping down to the upwave and downwave cylinders, then the behaviour at second- and third-order may be explained more succinctly in terms of this idealized first-order profile. Related to the first-order profile, these second- and third-order profiles shift upwave slightly with increasing wavelength (decreasing frequency).

At lower steepness along the centreline, the second-order profile has three peaks: at the edges of both cylinders and in the model centre. When compared with the corresponding first-order plots, the second-order profiles exhibit amplification at both first-order peaks and troughs, with second-order troughs at any maximum slopes of the first-order profile. It is important to note that the magnitude of the central peak seems reasonably constant at a value of 5 for most of the test frequencies. However, the second-order runup at the downwave cylinder's front face exhibits an increase with increasing wavelength to a maximum of 10 at $f=0.7277 \mathrm{~Hz}$ in figure 10 .

From observations of the lower-steepness case, the overall behaviour appears to be consistent with that observed previously in the $0^{\circ}$ heading tests, with third-order peaks corresponding to locations of peaks and maximum slopes of the first-order profile and third-order troughs at first order troughs and crest transition points (see above). Again, the magnitude of the third-order peaks is greater at the first-order maximum slopes than at the first-order peaks. At most of the frequencies, the predominance of third-order peaks at the first-order maximum slopes results in a third-order profile largely dominated by two peaks, one just upwave and the other just downwave of the central first order peak.

In the offset plots at lower-steepness, the second- and third-order profiles are, once again, more difficult to characterize in terms of those at first order. However, consistent patterns do exist and the global features adhere to the aforementioned trend of shifting upwave with increasing wavelength. At the highest frequency $(f=0.8000 \mathrm{~Hz}$ in figure 9), the second-order plot greatly resembles the first-order one, with peaks at the front and rear faces of the offset cylinder and gentle slopes away from the cylinder. As the frequency decreases, a trough appears downwave of the offset cylinder and, subsequently, a peak begins to develop further downwave as the trough shifts upwave at the lowest frequencies (for example at $f=0.7277 \mathrm{~Hz}$ in figure 10). The general behaviour at third order is characterized by a rise to a peak at the offset cylinder's front face. Downwave of the cylinder, the profile rises to a peak, drops to a trough $(x \approx 0.4 \mathrm{~m}$ at $f=0.8000 \mathrm{~Hz}$ in figure 9$)$, and then rises again to a peak. Again, these global features shift significantly forward with increasing wavelength; the aforementioned third-order trough, for example, shifts to $x \approx 0.3 \mathrm{~m}$ at $f=0.7277 \mathrm{~Hz}$ in figure 10 .

At the higher steepness, the second- and third-order profiles are quite different for several cases and are often difficult to characterize in terms of the first-order plots. At second order the higher steepness plots conform loosely to the above description at lower steepness with lower non-dimensionalized values at the peaks. However, as the wavelength increases in the centreline plots, and most notably at the lowest frequencies (for example at $f=0.7277 \mathrm{~Hz}$ in figure 10), the second-order profile changes into a trough at the upwave cylinder's rear face, followed by a steady rise to a central peak, 

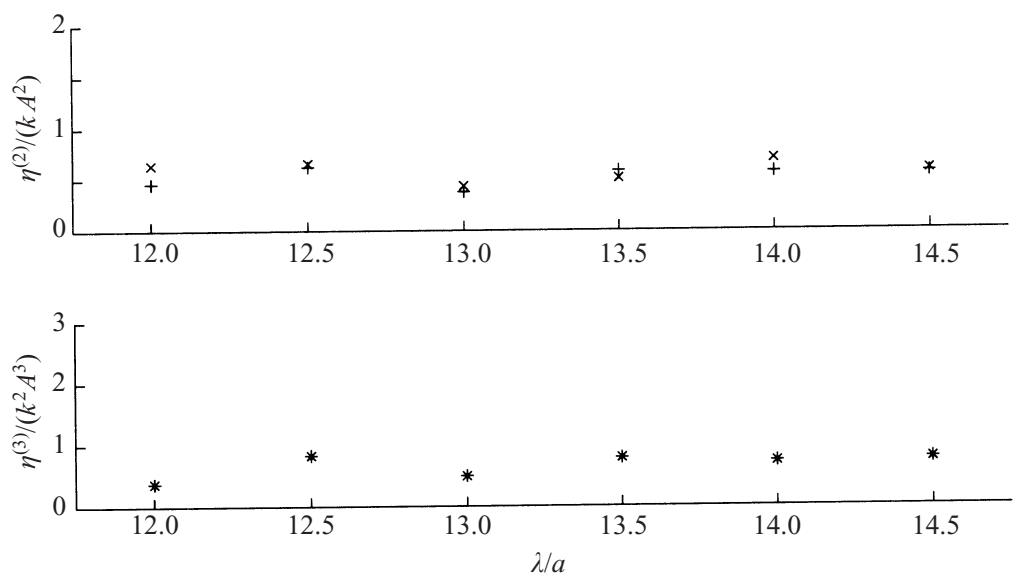

FIGURE 11. Second- and third-order amplitude versus non-dimensionalized wavelength; empty tank.

another trough, and a final rise to peak at the downwave cylinder's front face. The higher-steepness third-order profiles exhibit very low non-dimensionalized values and few significant features when compared to the corresponding lower-steepness cases. In the centreline plots, the primary feature of the third-order plots is a shallow peak just downwave of the model centre at $x \approx 0.2 \mathrm{~m}$. However, in the offset plots the profiles are nearly uniformly low in amplitude and not easily described in terms of peaks, troughs and slopes.

\subsubsection{Far-field radiation}

Using the data manipulation process described above, second- and third-order amplitudes were calculated for data taken at the reference gauge, located to the side of the model at $(x, y)=(-0.114 \mathrm{~m}, 3.500 \mathrm{~m})$. Any disturbance in the incident wave amplitude at this site can be taken as representative of the far-field radiation produced by the model. Calculated second- and third-order amplitudes are presented in figures 12 and 13 for the $0^{\circ}$ and $45^{\circ}$ heading cases, respectively. For comparison with these, figure 11 presents second- and third-order amplitudes of the undisturbed incident wave, computed as an average of amplitudes at each probe location during the empty tank tests. Non-dimensionalized second-order amplitude is shown in the top figure, with non-dimensionalized third-order amplitude in the bottom, and both are plotted against the incident wavelength to cylinder radius ratio.

These figures clearly show that the far-field higher-order components are significantly altered from what would be expected in the incident wave. The Stokes perturbation expansion in the undisturbed incident wave would produce non-dimensionalized values of $\frac{1}{2}$ and $\frac{3}{8}$ at second- and third-order, respectively. This corresponds well with the incident wave analysis of figure 11, although the third-order components are somewhat larger than the $\frac{3}{8}$ expected value. While significant differences do exist between the two heading cases, both far-field amplitude plots with the model present show amplification with respect to the empty tank results, and the lower-amplitude results tend to exceed those of the higher amplitude. Some exceptions to this do exist, such as the second-order amplitude at $\lambda / a=14$ for the $45^{\circ}$ heading, in which the higher-steepness result exceeds the lower.

Perhaps the most obvious observation from figures 12 and 13 is the contrast 

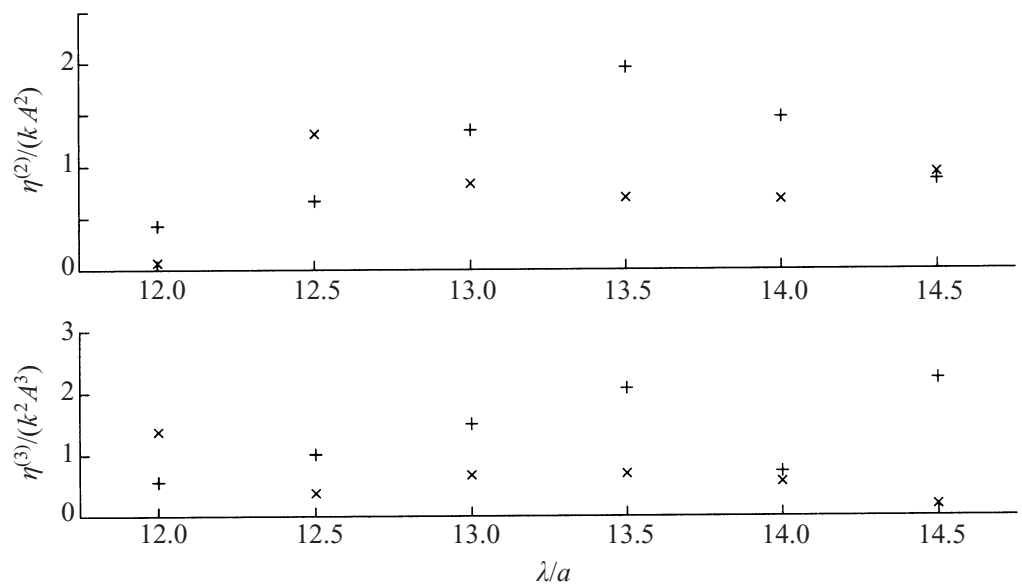

FIGURE 12. Second- and third-order amplitude versus non-dimensionalized wavelength; $\beta=0^{\circ}$.
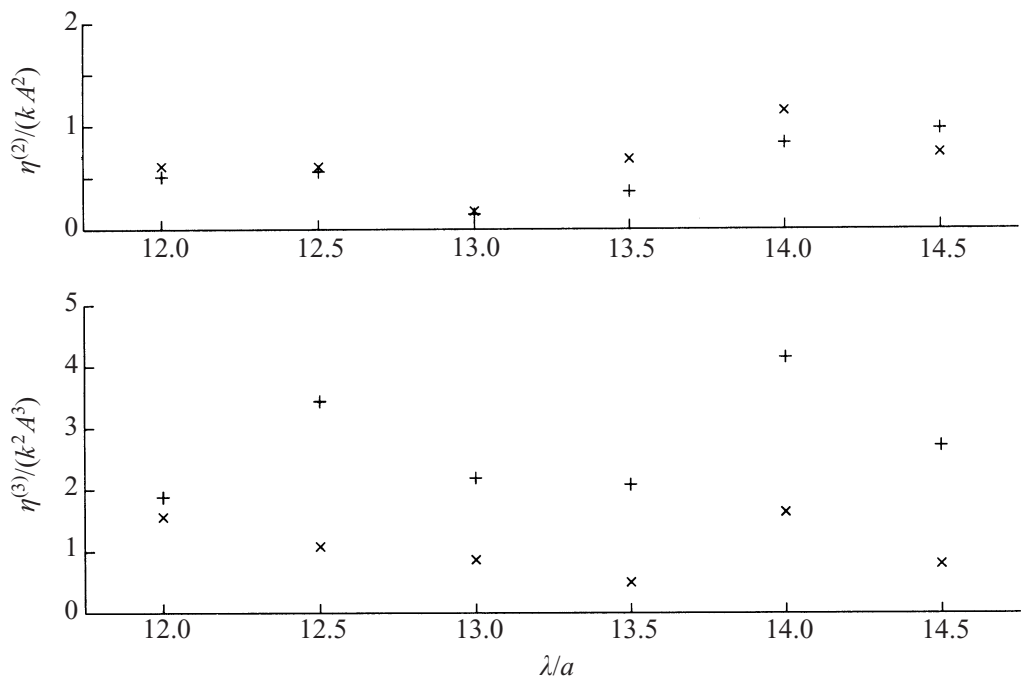

FIGURE 13. Second- and third-order amplitude versus non-dimensionalized wavelength; $\beta=45^{\circ}$.

between the predominance of second-order radiation in the $0^{\circ}$ heading case and third-order radiation in the $45^{\circ}$ heading case. In addition, significant variation of radiated amplitude occurs with change in incident wavelength. This is particularly of interest at $\lambda / a=13.5$, or $f=0.7542 \mathrm{~Hz}$, which corresponds to a near-trapping event (discussed further below). At this wavelength for the smaller steepness, the $0^{\circ}$ heading case exhibits a very clear maximum in far-field radiation at both second and third order, while the $45^{\circ}$ case exhibits a clear minimum in third-order radiation and a low value at second order. This is supported by video observations, as discussed above, in which more radiation was clearly visible for the $0^{\circ}$ heading case at this frequency in comparison to the $45^{\circ}$ heading case.

\subsection{Set-down and set-up}

The set-down or set-up observed beneath the model (computed as previously discussed) is presented here in figures 14 and 15 for heading $0^{\circ}$ and figures 16 and 17 
for heading $45^{\circ}$. In each figure part $(a)$ presents set-down or set-up as computed along the centre-line of the tank, whereas $(b)$ presents the offset set-down or set-up. Again, the ' $x$ ' and ' + ' symbols are used for the higher- and lower-amplitude cases, respectively, and vertical lines are drawn to denote the cylinder positions. The solid and dotted lines are drawn in $(a)$ only to aid the eye in distinguishing between the trends of the higher- and lower-amplitude cases.

\subsubsection{Heading $0^{\circ}$ : set-down and set-up observations}

In the centreline plots for the $0^{\circ}$ heading cases, there are two significant mean sea level peaks, one upwave of the model and a second near the model centre. This is indicative of significant set-up at these locations, between which are set-down troughs. These three global features occur at locations corresponding to the first-order upwelling sites discussed above in figures 7 and 8 and exhibit the same upwave shift behaviour with increasing wavelength and decreasing frequency. In the offset plots the mean sea level indicates set-up both at the front face of the upwave cylinder and between the upwave and downwave cylinders. In addition, large set-up is present at the runup sites on the downwave cylinder's front face for all frequencies and on the upwave cylinder's front face for $f=0.8000 \mathrm{~Hz}$ in figure 14 .

Some systematic differences appear to exist between the higher- and lower-steepness cases. At the four lowest frequencies (for example at $f=0.7277 \mathrm{~Hz}$ in figure 15), the lower-steepness downwave set-up in the centreline plot is significantly higher (in nondimensional terms) than that at the higher steepness. In contrast, at all frequencies apart from the highest at $f=0.8000 \mathrm{~Hz}$ in figure 14, the upwave centreline set-up is significantly higher for the higher-steepness case (again, figure 15 illustrates this).

\subsubsection{Heading $45^{\circ}$ : set-down and set-up observations}

For the $45^{\circ}$ heading, all of the mean sea level profiles indicate virtually no setdown. In contrast to the $0^{\circ}$ heading case analysed above, the global set-up features in the centreline plots correspond to those of the second-order free surface amplitude profiles as presented previously in figures 9 and 10. Like these amplitude profiles and most clearly visible in the lower steepness cases, these set-up plots show peaks at the inboard cylinder edges and at the model centre with two troughs upwave and downwave of the centre. With increasing wavelength, like results described above, the primary global feature of central set-up shifts upwave in the centreline plots. Similarly, with peaks at the offset cylinder's front and rear faces, the offset mean sea level profiles follow the general trends of the second-order amplitude profiles.

Again, some systematic differences are apparent between the lower- and highersteepness cases. At the lower steepness, the central peak is more clearly defined at all frequencies, whereas at some frequencies, notably $f=0.8000 \mathrm{~Hz}$ in figure 16 , the higher-steepness set-up profile yields almost no peak between the cylinders. In addition, the value at the downwave cylinder's front face is consistently greater for the lower-steepness cases.

The maximum set-up indicated occurs on the front face of the downwave cylinder at the lowest test frequency $(f=0.7277 \mathrm{~Hz}$ in figure 17) for the lower-steepness case. This corresponds to the maximum second-order runup observed previously at this frequency and steepness in figure 10. 
Wave diffraction. Part 1. Regular waves

(a)

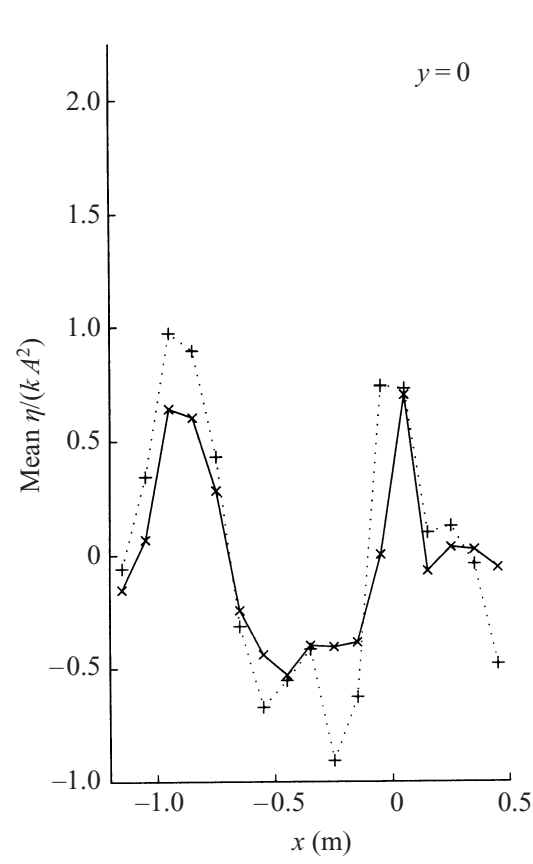

(b)

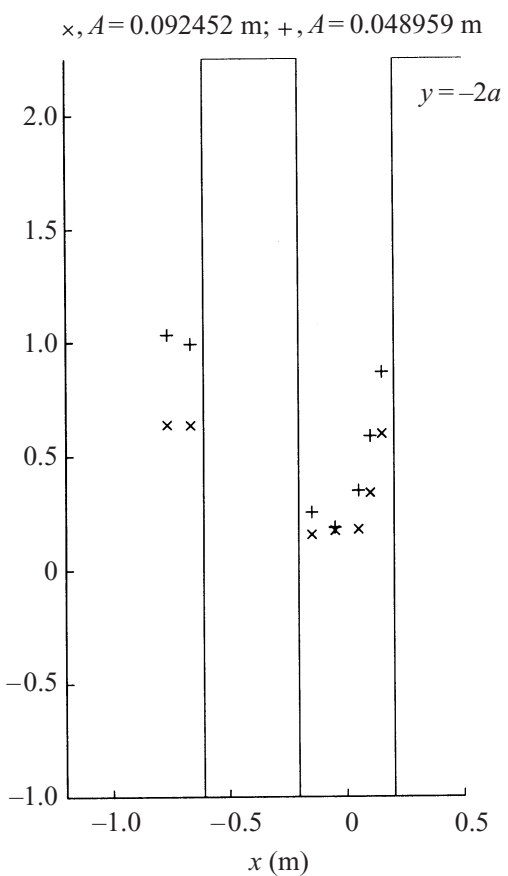

FIGURE 14. Set-down versus position; $\beta=0^{\circ}, f=0.8000 \mathrm{~Hz}$.

(a)

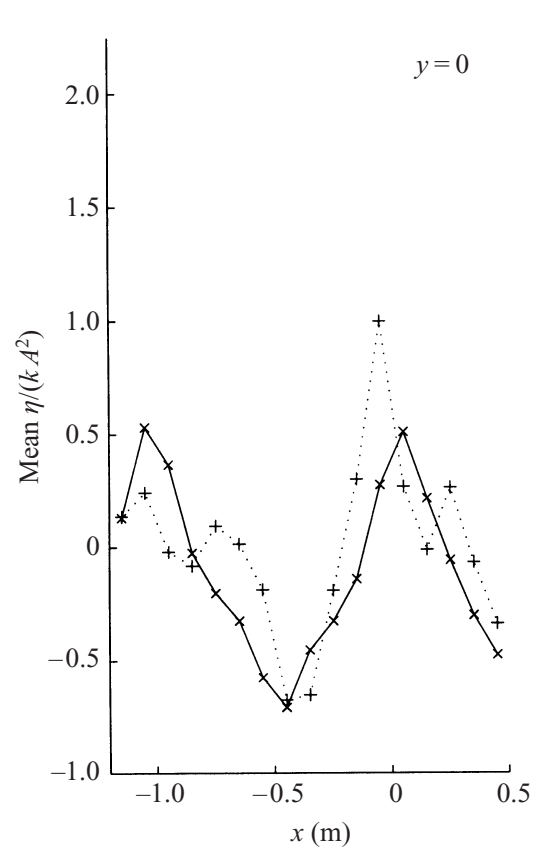

(b)

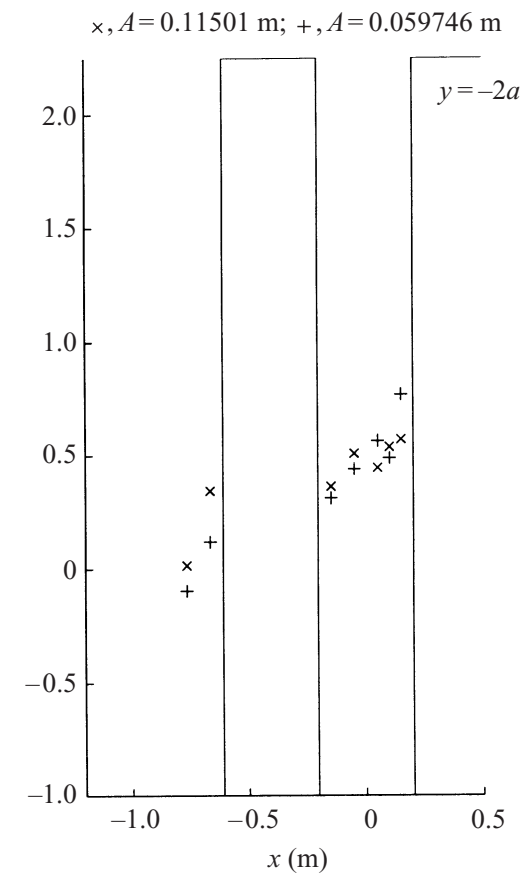

FIGURE 15. Set-down versus position; $\beta=0^{\circ}, f=0.7277 \mathrm{~Hz}$. 
(a)

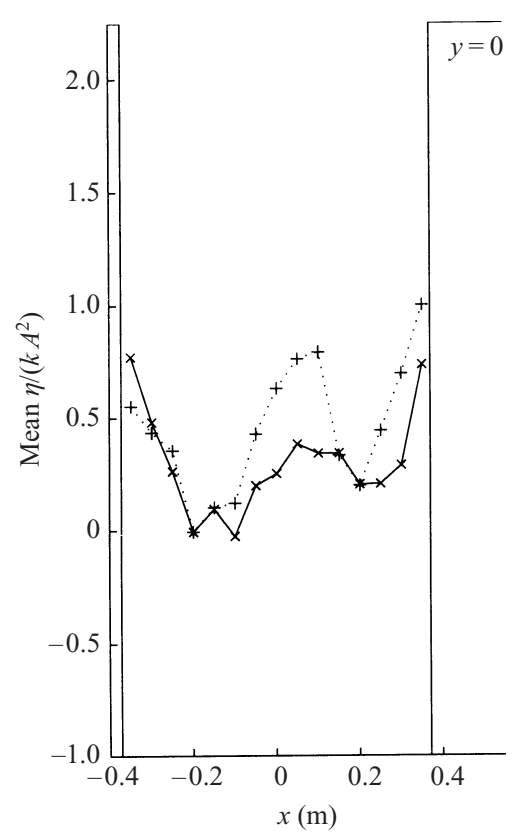

(b)

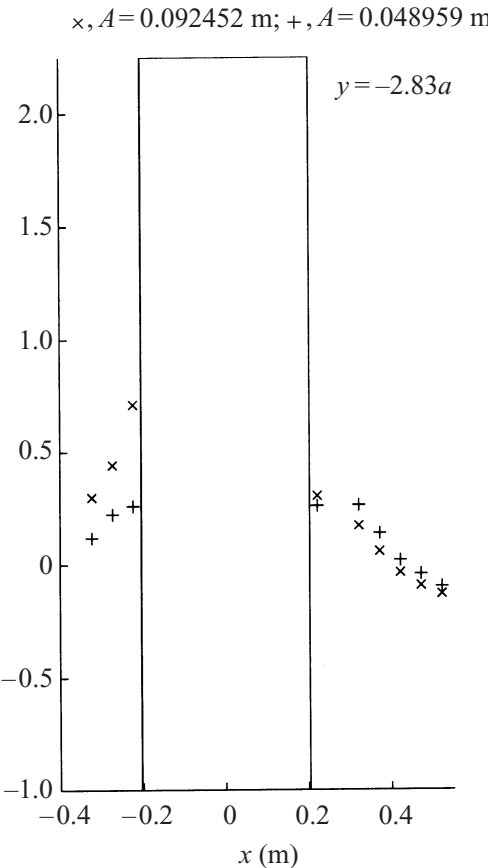

Figure 16. Set-down versus position; $\beta=45^{\circ}, f=0.8000 \mathrm{~Hz}$.

(a)

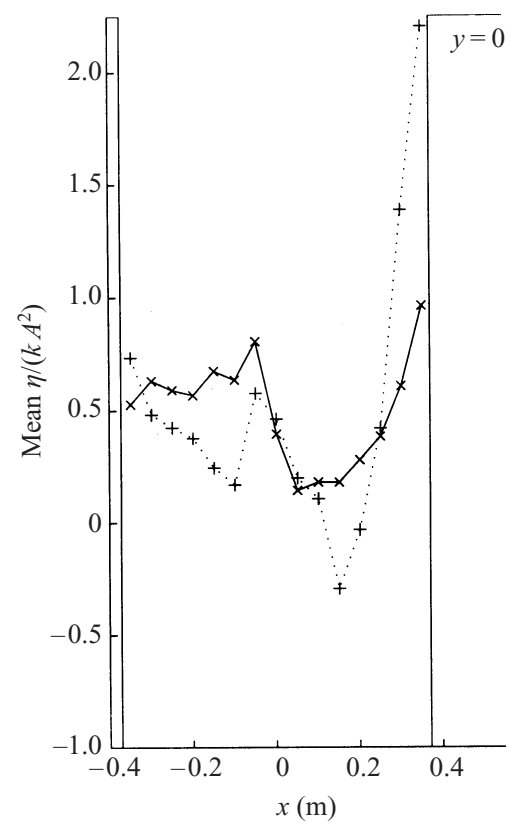

(b)

×, $A=0.11501 \mathrm{~m} ; \quad+, A=0.059746 \mathrm{~m}$

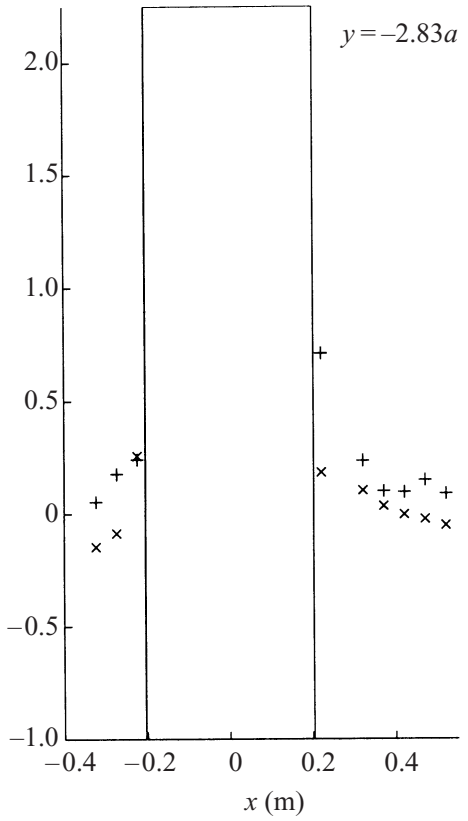

FIgURE 17. Set-down versus position; $\beta=45^{\circ}, f=0.7277 \mathrm{~Hz}$. 


\section{Discussion}

\subsection{Global features}

As predicted by first-order theory, the global features produced by the wave scattering shift steadily upwave with respect to the body as the incident wavelength is increased. This prediction has been verified in observations made from the video as well as plots of free surface amplitude and mean sea level profiles, as presented above. In addition, excellent agreement has been shown between first-order diffraction theory and the experimentally computed first-order amplitudes, particularly for incident waves of low steepness.

\subsection{Diffraction-induced breaking}

For the vast majority of the test cases, the first-order theoretical predictions were found to match more closely the lower-steepness incident wave cases. As linear diffraction theory is based on the potential flow assumption of small-amplitude incident waves, the result is not particularly surprising. However, significantly higher peaks in the non-dimensionalized amplitude profile are observed in some of the experiments. This could indicate some input of energy at the incident frequency from sources other than the first-order scattered wave, such as higher-order diffracted wave components. In addition, at higher steepness, the second- and third-order amplitude components were frequently far lower than those of the lower-amplitude cases. Given the assumed higher nonlinearity of steeper waves, this result is somewhat counter-intuitive.

One possible explanation for these observations is nonlinear breaking induced by diffraction. In this process, steep incident waves, already close to breaking for the steepest cases studied herein, interact with scattered waves from the structure. The resulting height of these superimposed waves is then too great to be supported given the incident wavelength. The incident and scattered waves then break and, in the generation of turbulence and foam, transfer energy to lower frequencies.

This process is observable to a certain extent in the videos produced during the testing. This diffraction-induced breaking could explain the aforementioned breaking stream observed at the upwelling site upwave of the model in the $0^{\circ}$ heading case. Moreover, it would directly explain the vast amounts of spray and foam generated at the model centre during the higher-steepness test cases.

\subsection{Effects at near-trapping frequency}

The geometry used in the $45^{\circ}$ heading case is identical to a case studied by Evans \& Porter (1997) and Malenica et al. (1999). The second-order investigation by Malenica et al. includes the near-trapping observed in the free surface amplitude profile for $k a=$ 0.468 . Malenica et al. observed that this non-dimensionalized frequency corresponds to near-trapping of the second order wave component (first-order near-trapping occurring at $k a=1.66$ as shown by Evans \& Porter). This $k a$ value is associated with a frequency which differs by only $0.64 \%$ from the frequency studied here, $f=0.7542 \mathrm{~Hz}$, which corresponds to $k a=0.465$.

Figure 18 displays a comparison of the experimental and theoretical results for first-order amplitude, second-order amplitude, and second-order set-down. As before, the solid line indicates the theoretical results, while the ' $x$ ' and ' + ' symbols represent the higher- and lower-steepness cases, respectively.

In the second-order plot, the global features of the free surface amplitude profile at near-trapping have been very well captured. In particular, the value of the central peak is very closely predicted, although the location of this predicted peak is approximately 

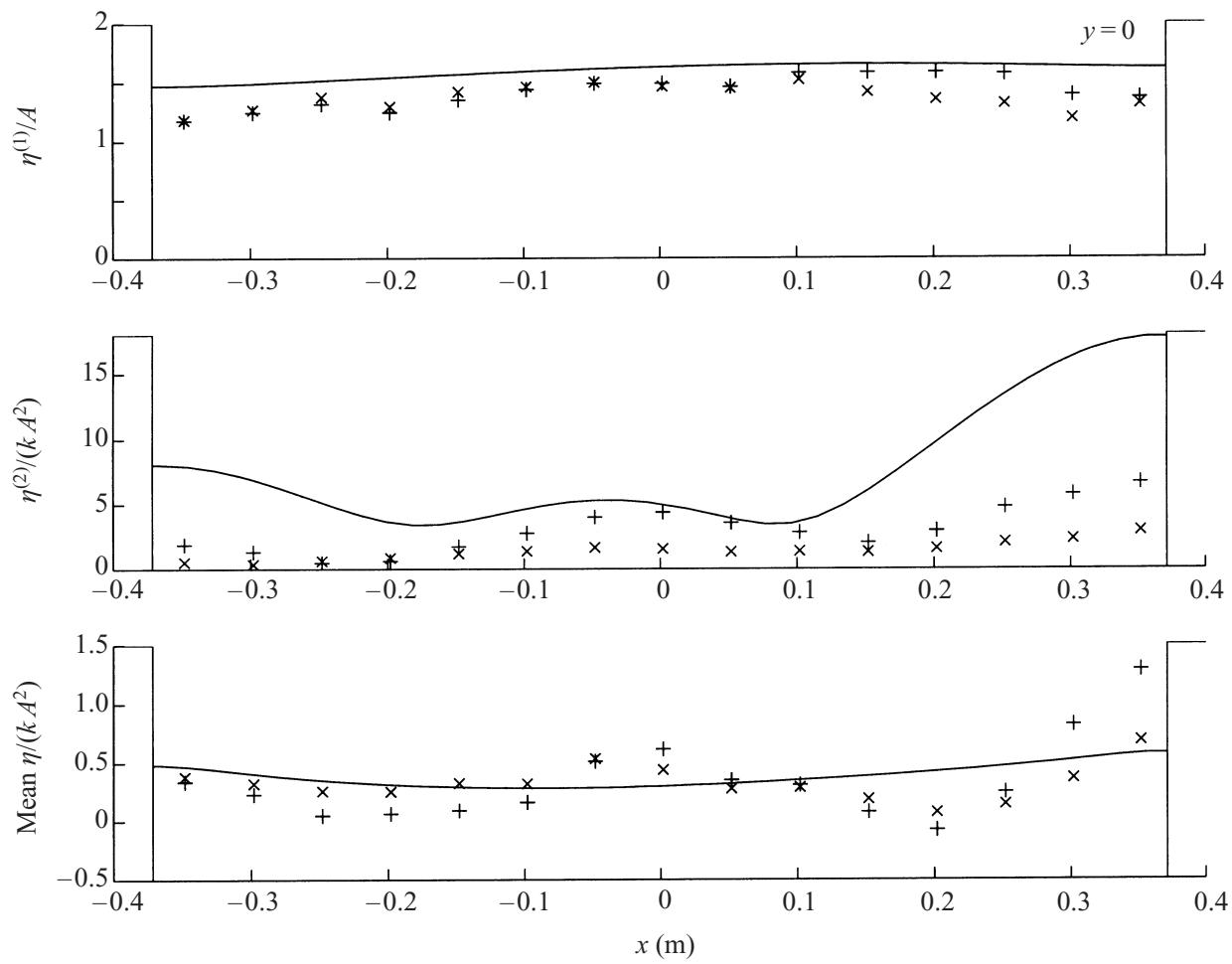

FIGURE 18. Theoretical and experimental results (for $\beta=45^{\circ}, f=0.7542 \mathrm{~Hz}$ ) at near-trapping; theoretical results reproduced with permission from Malenica et al. (1999).

$0.2 a(40 \mathrm{~mm})$ too far upwave. However, the runup values at the cylinder edges are significantly over predicted. Possible explanations for this are as follows:

diffraction-induced breaking disperses energy from this frequency component, as discussed above;

the data record, truncated to contain only 12 steady-state waves, was of insufficient length to fully saturate the second-order near-trapping event;

the near-trapping phenomenon is sensitive to small changes in $k a$ as observed by Malenica et al. (1999).

Figure 6(a) demonstrates the effect of the data record truncation at this frequency. In the upper plot, the pronounced second-order disturbance clearly grows with time well after the end of the analysed data section. However, it is not clear that this increasing disturbance is related to increased saturation of the second-order neartrapping mode as opposed to the build-up of reflected energy within the wave basin.

The theoretical profile of the second-order set-down and set-up poorly represents the global features of the mean sea level. Whereas the general magnitude and profile downwave of the upwave cylinder are well reproduced by the theory, the experimentally indicated peak at the model centre is not predicted. In addition, in the lower-steepness case, the large peak at the front face of the downwave cylinder is not well matched. However, it must be borne in mind that these theoretical results are limited to the second order and do not include potential contributions from higher-order components.

As previously mentioned in the discussion of far-field radiation, significant variation 
in second- and third-order radiated wave amplitude is observed at this secondorder near-trapping frequency for both headings. This frequency of $f=0.7452 \mathrm{~Hz}$ corresponds to the wavelength to cylinder radius ratio of $\lambda / a=13.5$ displayed in figures 12 and 13 . For the $0^{\circ}$ heading case (figure 12), excitation at this wavelength generates a clear maximum in both second- and third-order far-field radiation. This is in stark contrast to the clear minimum in third-order radiation and low amount of second-order radiation observed in the $45^{\circ}$ case (figure 13). In addition, the mean sea level profile at this frequency shows a significant drop near the model centre for the $0^{\circ}$ heading as can be observed in figures 14 and 15 . This is particularly clear for the lower steepness case relative to the set-down at other frequencies. However, the $45^{\circ}$ heading mean sea level profile shows a set-up at the centre that is reasonably consistent with that at other frequencies, such as those presented in figures 16 and 17.

These results are indicative of two widely differing responses at the second-order near-trapping frequency for the two test geometries:

for the $0^{\circ}$ heading case, far-field radiation is maximized and is accompanied by a relative decrease in local mean sea level at the model centre;

for the $45^{\circ}$ heading case, far-field radiation is minimized while a set-up is maintained at the model centre.

This suggests general tendencies for energy dissipation and radial flow from the centre for the $0^{\circ}$ heading cases at the near-trapping frequency, with a contrasting tendency to trap energy and fluid at the centre for the $45^{\circ}$ heading. This echoes observations made from the video data, in which local radiation and fluid propagating from the model centre appeared far more significant in the $0^{\circ}$ heading cases in relation to the $45^{\circ}$ heading cases. It is an indication that the second-order near-trapping mode at this frequency has symmetry about the diagonals of the square array, rather than about the bisectors of the sides (Evans \& Porter have discussed the symmetries of the first-order near-trapping modes). Finally, given the sheltered geometry of the $45^{\circ}$ heading case, this result is somewhat intuitive.

\section{Conclusions}

The main conclusions can be summarized as follows:

(a) Contour plots of the diffracted free surface amplitude for regular waves interacting with four-cylinder configurations have been produced through linear diffraction theory. These have been shown to provide excellent predictions of visually observable regions of enhanced wave activity, such as upwelling between and runup adjacent to the cylinders.

(b) Linear diffraction theory has been shown to be an excellent model of the first-order free surface elevation response, particularly for incident waves of low steepness.

(c) As predicted by linear theory, it has been shown experimentally that global features present in first-order amplitude profiles shift consistently upwave with increases in incident wavelength. This has also been observed for experimentally computed profiles of local mean sea level, a second-order quantity.

(d) Through both video observations and data analysis, highly nonlinear wave breaking has been identified as induced by diffraction for high-steepness incident waves.

(e) With local wave elevation enhancement, increased mean sea level (set-up) at the model centre, and diminished far-field radiation, near-trapping has been observed 
experimentally for $k a$ within $0.64 \%$ of a second-order near-trapping event (Malenica et al. 1999) for the $45^{\circ}$ heading tests.

$(f)$ However, the experimentally measured second-order contributions to free surface elevation were significantly lower than predicted by the semi-analytical diffraction theory of Malenica et al. (1999). This discrepancy may be attributed to either the aforementioned diffraction-induced breaking of steep incident waves, or the insufficient length of the data record, or the sensitivity of the near-trapping phenomenon to small changes in $k a$.

$(g)$ For this near-trapping frequency, corresponding tests at heading $0^{\circ}$ have yielded high far-field radiation and decreased mean sea level (set-down) near the model centre. As observed visually for this less sheltered geometry, this is indicative of increased wave scattering in the far field associated with radial flow from the model centre.

The authors acknowledge the support of HR Wallingford and Dr Roger Wilkinson, the former Offshore Group Manager at HR, for the time in their facility and the construction of the model. The first author was supported through a British Marshall Scholarship from the Marshall Commemoration Commission. The authors are grateful for the constructive comments of the referees.

\section{Appendix. Linear diffraction theory for regular waves}

Linear diffraction theory for regular waves is presented here with some slight modifications to the original derivation of Linton \& Evans (1990), which may be referred to for explanation of undefined terms or solution stages. For the first-order interaction of regular waves with arrays of $N$ bottom-mounted vertical circular cylinders, the velocity potential may be assumed to be of the form

$$
\Phi(x, y, z, t)=\operatorname{Re}\left\{\phi(x, y) f_{0}(z) \mathrm{e}^{-\mathrm{i} \omega t}\right\},
$$

where the depth attenuation function $f_{0}(z)$ is defined by

$$
f_{0}(z)=-\frac{\mathrm{i} g A}{\omega} \frac{\cosh k(z+d)}{\cosh k d} .
$$

In the case of multiple cylinders, the wave scattered by cylinder $j$ may be expressed in polar coordinates centred on cylinder $j$ (which has radius $a_{j}$ ). Thus:

$$
\phi_{S}^{j}=\sum_{n=-\infty}^{\infty} A_{n}^{j} Z_{n}^{j} H_{n}\left(k r_{j}\right) \mathrm{e}^{\mathrm{i} n \theta_{j}},
$$

for some set of complex coefficients $A_{n}^{j}$ and with $Z_{n}^{j}=J_{n}^{\prime}\left(k a_{j}\right)$. This definition of $Z$ differs from that presented by Linton \& Evans (1990) and produces a better conditioned matrix for the solution of the coefficients. Note that $H_{n}$ and $J_{n}^{\prime}$ are Hankel functions and the first derivative of the Bessel function of the first kind of order $n$.

Following from the above, the total potential is then expressed using Graf's addition theorem as

$$
\begin{aligned}
\phi & =\phi_{I}+\sum_{j=1}^{N} \phi_{S}^{j} \\
& =I_{j} \sum_{n=-\infty}^{\infty} J_{n}\left(k r_{j}\right) \mathrm{e}^{\mathrm{i} n\left(\pi / 2-\theta_{j}+\beta\right)}+\sum_{j=1}^{N} \sum_{n=-\infty}^{\infty} A_{n}^{j} Z_{n}^{j} H_{n}\left(k r_{j}\right) \mathrm{e}^{\mathrm{i} n \theta_{j}},
\end{aligned}
$$


where $\beta$ is the wave heading measured with respect to the $x$-axis.

The coefficients $A_{m}^{k}$ are obtained from

$$
\begin{array}{r}
A_{m}^{k} H_{m}^{\prime}\left(k a_{k}\right)+\sum_{j=1, j \neq k}^{N} \sum_{n=-\infty}^{\infty} A_{n}^{j} Z_{n}^{j} H_{n-m}\left(k R_{j k}\right) \mathrm{e}^{\mathrm{i}(n-m) \alpha_{j k}}=-I_{k} \mathrm{e}^{\mathrm{i} m(\pi / 2-\beta)} \\
\text { for } k=1, \ldots, N, \quad \text { and } \quad-\infty<m<\infty .
\end{array}
$$

This infinite system of equations may be truncated from $-M<m<M$ to produce an $N(2 M+1)$ system of equations in $N(2 M+1)$ unknowns.

The free surface elevation may be found as

$$
\eta(x, y, t)=\operatorname{Re}\left\{A \phi(x, y) \mathrm{e}^{-\mathrm{i} \omega t}\right\} .
$$

From this, the local modification to the incident wave amplitude due to diffraction is

$$
\frac{|\eta(x, y, t)|}{A}=|\phi(x, y)| \text {. }
$$

As the non-dimensionalized component of the velocity potential with depth and time dependence removed, this variable $\phi(x, y)$ may be referred as the diffraction coefficient to further emphasize its modification of the incident wave amplitude.

\section{REFERENCES}

Celebi, M. S., Kim, M. H. \& BeCK, R. F. 1998 Fully non-linear 3D numerical wave tank simulation. J. Ship Res. 42, 33-45.

EAtock TAYLOR, R. \& Wu, G. X. 1997 Interaction of steep waves with offshore structures. Phil. Trans. R. Soc. Lond. A 355, 593-605.

Evans, D. V., Levitin, M. \& Vassiliev, D. 1994 Existence theorems for trapped modes. J. Fluid Mech. 261, 21-31.

Evans, D. V. \& Linton, C. M. 1991 Trapped modes in open channels. J. Fluid Mech. 225, 153-175.

Evans, D. V. \& PORTER, R. 1997 Near-trapping of waves by circular arrays of vertical cylinders. Appl. Ocean Res. 19, 83-99.

FERRANT, P. 1996 Simulation of strongly non-linear wave generation and wave-body interactions using a 3D MEL model. Proc. 21st ONR Symp. on Naval Hydrodynamics, Trondheim, Norway. Washington DC: National Academy Press.

Huang, J. B. \& Eatock TAYloR, R. 1996 Second order interaction between waves and multiple bottom-mounted vertical circular cylinders. Proc. 11th Workshop on Water Waves and Floating Bodies, Hamburg. (ed. V. Bertram). Institut für Schiffbau

Huseby, M. \& Grue, J. 1998 An experimental investigation of higher harmonic forces on a vertical cylinder in long waves. Proc. 13th Intl Workshop on Water Waves and Floating Bodies, Alphen aan den Rijn (ed. A. J. Hermans), pp. 43-46. Delft University of Technology.

JoNES, D. S. 1953 The eigenvalues of $\nabla^{2} \boldsymbol{u}+\lambda u=0$ when the boundary conditions are given on semi-infinite domains. Proc. Camb. Phil. Soc. 49, 668-684.

KIM, M. \& YUE, D. K. P. 1989 The complete second-order diffraction solution for an axisymmetric body. Part 1. Monochromatic incident waves. J. Fluid Mech. 205, 235-264.

KRIEBEL, D. L. 1990 Nonlinear wave interaction with a vertical circular cylinder. Part 1. Diffraction theory. Ocean Engng 17, 345-377.

Kriebel, D. L. 1992 Nonlinear wave interaction with a vertical circular cylinder. Part 2. Wave Runup. Ocean Engng 19, 75-99.

Linton, C. M. \& Evans, D. V. 1990 The interaction of waves with arrays of vertical circular cylinders. J. Fluid Mech. 215, 549-569.

Ma, Q. W., Wu, G. X. \& Eatock Taylor, R. 2001 Finite element simulation of fully non-linear interaction between a vertical cylinder and steep waves-part I: Methodology and numerical procedure. Intl J. Numer. Meth. Engng (in press). 


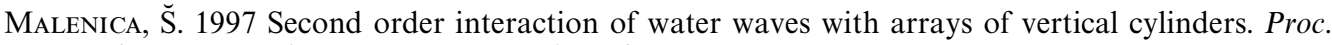
2nd Congress of Croatian Society of Mechanics, Supetar, Croatia, pp. 599-606.

Malenica, S̆., Eatock Taylor, R. \& Huang, J. B. 1999 Second order water wave diffraction by an array of vertical cylinders. J. Fluid Mech. 390, 349-373.

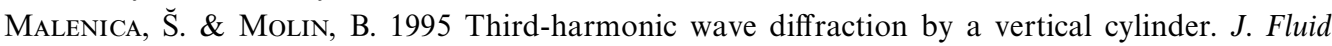
Mech. 302, 203-229.

Maniar, H. D. \& Newman, J. N. 1997 Wave diffraction by a long array of cylinders. J. Fluid Mech. 339, 309-330.

MeI, C. C. 1983 The Applied Dynamics of Ocean Surface Waves, 2nd Edn. John Wiley \& Sons.

MoE, G. (Ed.) 1993 Vertical resonant motions of TLPs. Final Report. NTH Rep, R-1-93.

OHL, C. O. G. 1999 Free surface disturbances and nonlinear runup around offshore structures. DPhil thesis, University of Oxford.

Ohl, C. O. G., Taylor, P. H., Eatock Taylor, R. \& Borthwick, A. G. L. 2001 Diffraction by a cylinder array. Part 2. Irregular waves J. Fluid Mech. 442, 33-66.

Owen, D. G. \& Padilla Perez, I. A. 1987 Experimental investigation of upwelling and interaction effects within blocked structures. Final Report, Programme of Research into Dynamics of Compliant Structures 1985-87, Vol. I.

Stokes, G. G. 1846 Report on recent researches in hydrodynamics. Br. Ass. Rept. 1846; see also Collected Papers, Vol. I, p. 167.

Swan, C., TAYlor, P. H. \& VAn LAngen, H. 1997 Observations of wave-structure interaction for a multi-legged concrete platform. Appl. Ocean Res. 19, 309-327.

Ursell, F. 1951 Trapping modes in the theory of surface waves. Proc. Camb. Phil. Soc. 47, 347-358.

Yeung, R. W. 1982 Numerical methods in free surface flows. Ann. Rev. Fluid Mech. 14, 395-442. 